\title{
Fundamental Solution for a Degenerate Hyperbolic Operator in Gevrey Classes
}

\author{
By \\ Kenzo ShINKAI* and Kazuo Taniguchi*
}

\section{Imtroduction}

In [9] Ivrii proved that the Cauchy problem of a degenerate hyperbolic operator

$$
D_{t}^{2}-t^{2 l} D_{x}^{2}+a t^{k} D_{x}
$$

with $l-1>k \geqq 1$ is well-posed in a Gevrey class of order $\kappa$ if and only if $1 \leqq \kappa<(2 l-k) /(l-k-1)$ and the Cauchy problem of

$$
D_{t}^{2}-x^{2 l^{\prime}} D_{x}^{2}+a x^{k^{\prime}} D_{x}
$$

with $l^{\prime}>k^{\prime} \geqq 0$ is well-posed in a Gevrey class of order $\kappa$ if and only if $1 \leqq \kappa<\left(2 l^{\prime}-k^{\prime}\right) /\left(l^{\prime}-k^{\prime}\right)$. Combining these degeneracy we study, in the present paper, second order hyperbolic operators including

$$
D_{t}^{2}-t^{2 l} x^{2 l^{\prime}} D_{x}^{2}+a t^{k} x^{k^{\prime}} D_{x}
$$

as a prototype. Let $\sigma$ be a constant

$$
\sigma=\max \left((l-k-1) /(2 l-k),\left(l^{\prime}-k^{\prime}\right) /\left(2 l^{\prime}-k^{\prime}\right)\right) \quad(<1 / 2)
$$

and $\sigma^{\prime}$ be a constant satisfying

$$
\sigma<\sigma^{\prime}<1 / \kappa, \quad \sigma^{\prime} \geqq\left(1+\left(l^{\prime}-1\right) \sigma\right) /\left(l^{\prime} \kappa-l^{\prime}+1\right)
$$

for $\kappa$ such that $2 \leqq \kappa<1 / \sigma$. We construct the fundamental solution for the Cauchy problem and show that it is estimated by $C \exp \left(C_{1}\langle\xi\rangle^{\sigma^{\prime}}\right)$. Then we can obtain not only the well-posedness of the Cauchy problem but also the branching properties for the propagation of Gevrey singularities. We note that

Communicated by S. Matsuura, December 24, 1987.

1991 Mathematics Subject Classifications: 35L80 secondary 47G30

* Department of Mathematics, University of Osaka Prefecture, Sakai, Osaka 591, Japan. 
Itoh and Uryu [8] have already proved that (3) is well-posed in a Gevrey class of order $\kappa$ with $1 \leqq \kappa<1 / \sigma$ for $\sigma$ defined by (4).

The operator treated in this paper is

$$
\begin{aligned}
L= & D_{t}^{2}-t^{2 l} g(x)^{2 l^{\prime}} \sum_{j, j^{\prime}=1}^{n} a_{j, j^{\prime}}(t, x) D_{x_{j}} D_{x_{j^{\prime}}} \\
& +t^{k} g(x)^{k^{\prime}} \sum_{j=1}^{n} a_{j}(t, x) D_{x_{j}}+c(t, x) \quad \text { on }[0, T] .
\end{aligned}
$$

We assume the following:

(A-1) $l-1 \geqq k \geqq 0, l^{\prime} \geqq k^{\prime} \geqq 1$ and $l^{\prime} \geqq 2$.

(A-2) $\kappa \geqq 2$ and $\kappa \sigma<1$ with $\sigma$ in (4).

$(A-3)$ The function $g(x)$ belongs to a Gevrey class of order $\kappa$ with a uniform estimate

$$
\left|D_{x}^{\alpha} g(x)\right| \leqq C M^{-|\alpha|} \alpha !^{\kappa} \quad \text { for all } x \in \mathbb{R}^{n} .
$$

The coefficients $a_{j, j^{\prime}}(t, x), a_{j}(t, x)$ and $c(t, x)$ are analytic in $t$ and of a Gevrey class of order $\kappa$ in $x$ with a uniform estimate (7). that

(A-4) $a_{j, j^{\prime}}(t, x)$ are real-valued and there exists a positive constant $\mathbb{C}$ such

$$
\sum_{j, j^{\prime}} a_{j, j^{\prime}}(t, x) \xi_{j} \xi_{j^{\prime}} \geqq C|\xi|^{2} \quad \text { for all } \quad(t, x) \in[0, T] \times \mathbb{R}_{x}^{n} .
$$

Then, we have

Theorem H。 $_{\text {. We assume }}(\mathbb{A}-1)-(\mathbb{A}-4)$. Set $\rho=1-(1-\sigma) / l^{\prime}$. Then, for a small $T_{0}(\leqq T)$ we can construct the fundamental solution $\mathbb{E}(t, s)$ for the Cauchy problem

$$
\begin{cases}L u=0 & \text { on }\left[s, T_{0}\right], \\ u(s)=0, & \partial_{t} u(s)=u_{0}\end{cases}
$$

with $s \in\left[0, T_{0}\right)$ in the form

$$
\mathbb{E}(t, s)=\sum_{ \pm} \mathbb{I}_{\phi_{ \pm}}(t, s) E_{ \pm}(t, s)+E_{0}(t, s)+E_{\infty}(t, s) .
$$

Here, $I_{\phi_{ \pm}}(t, s)$ are Fourier integral operators with the symbol 1 , and $E_{j}(t, s), j=0$, ,$\pm \infty$, are pseudo-differential operators with symbols $e_{j}(t, s ; x, \xi)$ satisfying

$$
\begin{aligned}
& \left|e_{ \pm(\beta)}^{(\alpha)}(t, s ; x, \xi)\right| \leqq C M^{-|\alpha+\beta|}\left((\alpha+\beta) !^{\kappa}+(\alpha+\beta) !^{\kappa \rho}\langle\xi\rangle^{(1-\rho)|\alpha+\beta|}\right) \\
& \times\langle\xi\rangle^{-|\alpha|} \exp \left(C_{1}\langle\xi\rangle^{\sigma^{\prime}}\right), \\
& \left|e_{0(\beta)}^{(\alpha)}(t, s ; x, \xi)\right| \leqq C M^{-|\alpha+\beta|}\left((\alpha+\beta) !^{\kappa}+(\alpha+\beta) !^{\kappa \rho}\langle\xi\rangle^{(1-\rho)|\alpha+\beta|}\right) \text {, } \\
& \times\langle\xi\rangle^{-|\alpha|} \exp \left(C_{1}\langle\xi\rangle^{\sigma^{\prime}}-\varepsilon_{1} t^{l+1}|g(x)|^{l^{\prime}}\langle\xi\rangle^{1-\sigma}\right),
\end{aligned}
$$


for a positive constant $\varepsilon_{1}$ and the constant $\sigma^{\prime}$ satisfying (5). Moreover, for any multi-index $\alpha$ there exists a constant $C_{\alpha}$ such that

$$
\left|e_{\infty(\beta)}^{(\alpha)}(t, s ; x, \xi)\right| \leqq C_{\alpha} M^{-|\beta|} \beta !^{\kappa} \exp \left(-\varepsilon_{2}\langle\xi\rangle^{1 / \kappa}\right)
$$

for a positive constant $\varepsilon_{2}$.

We remark that the condition $\sigma^{\prime} \geqq\left(1+\left(l^{\prime}-1\right) \sigma\right) /\left(l^{\prime} \kappa-l^{\prime}+1\right)$ in $(5)$ and the analyticity of the coefficients of (6) enable us to construct the fundamental solution of (8) as a sum of Fourier integral operators with only simple phase functions as in (9).

Combining this theorem with discussion in [18], we obtain the branching properties as follows. Let $\mathrm{WF}_{G(\kappa)}(u)$ be the Gevrey wave front set of a ultradistribution $u$ (cf. [7], [23]), and, setting

$$
\lambda_{ \pm}(t, x, \xi)= \pm t^{l} g(x)^{l^{\prime}}\left\{\sum_{j, j^{\prime}} a_{j, j^{\prime}}(t, x) \xi_{j} \xi_{j^{\prime}}\right\}^{1 / 2}
$$

let $\left\{q^{ \pm}, p^{ \pm}\right\}(t, s ; x, \xi)$ be the solution of

$$
\left\{\begin{array}{l}
\frac{d q^{ \pm}}{d t}=-\nabla_{\xi} \lambda_{ \pm}\left(t, q^{ \pm}, p^{ \pm}\right), \quad \frac{d p^{ \pm}}{d t}=\nabla_{x} \lambda_{ \pm}\left(t, q^{ \pm}, p^{ \pm}\right) \quad\left(s \leqq t \leqq T_{0}\right), \\
\left\{q^{ \pm}, p^{ \pm}\right\}_{\mid t=s}=(y, \eta)
\end{array}\right.
$$

and $\left\{\tilde{q}^{ \pm}, \tilde{p}^{ \pm}\right\}(t, s ; y, \eta)$ be the solution of

$$
\left\{\begin{array}{l}
\frac{d \tilde{q}^{ \pm}}{d t}=-\nabla_{\xi} \lambda_{ \pm}\left(t, \tilde{q}^{ \pm}, \tilde{p}^{ \pm}\right), \quad \frac{d \tilde{p}^{ \pm}}{d t}=\nabla_{x} \lambda_{ \pm}\left(t, \tilde{q}^{ \pm}, \tilde{p}^{ \pm}\right) \quad\left(0 \leqq t \leqq T_{0}\right), \\
\left\{\tilde{q}^{ \pm}, \tilde{p}^{ \pm}\right\}_{\mid t=0}=\left\{q^{\mp}, p^{\mp}\right\}(0, s ; y, \eta) .
\end{array}\right.
$$

Theorem 2. Consider a Cauchy problem (8) with $s<0$. Then we have, when $t>0$, for a solution $u(t)$ of (8)

$$
\mathrm{WF}_{G(\kappa)}(u(t)) \subset \Gamma_{+}(t) \cup \Gamma_{-}(t) \cup \tilde{\Gamma}_{+}(t) \cup \tilde{\Gamma}_{-}(t) \cup \Gamma_{0}(t),
$$

where

$$
\begin{aligned}
& \Gamma_{ \pm}(t)=\left\{\left(q^{ \pm}(t, s ; y, \eta), p^{ \pm}(t, s ; y, \eta)\right) ;(y, \eta) \in \mathrm{WF}_{G(\kappa)}\left(u_{0}\right),|\eta| \gg 1\right\}, \\
& \tilde{\Gamma}_{ \pm}(t)=\left\{\left(\tilde{q}^{ \pm}(t, s ; y, \eta), \tilde{p}^{ \pm}(t, s ; y, \eta)\right) ;(y, \eta) \in \mathrm{WF}_{G(\kappa)}\left(u_{0}\right),|\eta| \gg 1\right\}
\end{aligned}
$$

and

$$
\Gamma_{0}(t)=\left\{(y, \eta) ;(y, \eta) \in \mathrm{WF}_{G(\kappa)}\left(u_{0}\right), g(y)=0\right\}
$$

This theorem corresponds to the branching property for the $C^{\infty}$-case, that is, for the Cauchy problem of the operator (1) with $k=l-1$ (see [1], [24] and [18]). We note that the first author gave $\mathrm{WF}_{G(\kappa)}(u(t))$ exactly by using the 
exact form of the fundamental solution for the operator (1) with $l-1>k \geqq 0$ (see [19], [20]). In $(\mathbb{A}-2)-(\mathbb{A}-3)$ we assumed $\kappa \geqq 2$. But, in case $1<\kappa<2$, the problem (8) for (6) is always $\gamma^{(\kappa)}$-well-posed for any lower order terms and in this case the propagation of singularities (13) for a solution of (8) is obtained in [15].

The outline of this paper is as follows. In Secions 1 and 2 we give caluculus of pseudo-differential operators and Fourier integral operators. In Section 3 we introduce symbol classes of pseudo-differential operators and give lemmas. In Section 4 we reduce the Cauchy problem (8) to the Cauchy problem of a perfectly diagonalized system and state Theorern 3, which is the version of Theorem 1 for a hyperbolic system. Sections 5 and 6 are devoted to the proof of Theorem 3.

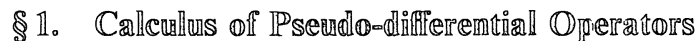

Throughout this section the real numbers $\rho, \delta$ and $\kappa$ always satisfy $0 \leqq$ $\delta \leqq \rho \leqq 1, \delta<1, \kappa(1-\delta) \geqq 1, \kappa \rho \geqq 1$ and $\kappa>1$.

Defimitiom $\mathbb{1}_{0} \mathbb{1}_{\text {. }}$ i) Let $w(\theta)$ be a positive and non-decreasing function in $[1, \infty)$ or a function of the type $\theta^{m}$ for a real $m$. We say that a symbol $p(x, \xi)$ belongs to a class $\mathbb{S}_{\rho, \delta, G(\kappa)}[w]$ if $p(x, \xi)$ satisfies

$$
\begin{aligned}
\left|p_{(\beta)}^{(\alpha)}(x, \xi)\right| \leqq & C M^{-|\alpha+\beta|}\left(\alpha !^{\kappa}+\alpha !^{\kappa \rho}\langle\xi\rangle^{(1-\rho)|c|}\right) \\
& \times\left(\beta !^{\kappa}+\beta !^{\kappa(1-\delta)}\langle\xi\rangle^{\delta|\beta|}\right)\langle\xi\rangle^{-|\alpha|} w(\langle\xi\rangle)
\end{aligned}
$$

for all $x$ and $\xi$, where $p_{(\beta)}^{(\alpha)}=\partial_{\xi}^{\alpha}\left(-i \partial_{x}\right)^{\beta} p$. (cf. [14], [10]). We say that inf $\{C$ of $(1.1)\}$ is a formal norm of $p(x, \xi)$ and denote it by $\|p ; \mathbb{M}\|$.

ii) Let $w(\theta)$ be the same as above. We say that a symbol $p(x, \xi)$ belongs to a class $S W F_{1, \delta, G(\kappa)}[w]$ if $p(x, \xi)$ belongs to a class $\mathbb{S}_{1, \delta, G(\kappa)}[w]$ and there exists a formal sum $\sum p_{j}(x, \xi)$ of symbols $p_{j}(x, \xi)$ satisfying

$$
\begin{aligned}
\left|p_{j(\beta)}^{(\alpha)}(x, \xi)\right| \leqq & C M^{-(|\alpha|+|\beta|+j)} \alpha ! \\
& \times\left((|\beta|+j) !^{\kappa}+(|\beta|+j) !^{\kappa(1-\delta)}\langle\xi\rangle^{\delta(|\beta|+j)}\right) \\
& \times\langle\xi\rangle^{-j-|\alpha|} w(\langle\xi\rangle) \quad \text { for } \quad|\xi| \geqq c
\end{aligned}
$$

with a constant $c(\geqq 1)$ and

$$
\begin{aligned}
\mid \partial_{\xi}^{\alpha} \partial_{x}^{\beta}(p(x, \xi)- & \left.\sum_{j=0}^{N-1} p_{j}(x, \xi)\right) \mid \leqq C M^{-(|\alpha|+|\beta|+N)} \alpha ! \\
& \times\left((|\beta|+N) !^{\kappa}+(|\beta|+N) !^{\xi(1-\delta)}\langle\xi\rangle^{\delta(|\beta|+N)}\right) \\
& \times\langle\xi\rangle^{-|\alpha|-N} w(\langle\xi\rangle) \quad \text { for } \quad|\xi| \geqq c(|\alpha|+N)^{\kappa}
\end{aligned}
$$


for any $N$. In this case we say that the formal sum $\sum p_{j}(x, \xi)$ is the formal symbol associated with $p(x, \xi)$. As in i) we say that $\inf \{C$ of $(1.1)-(1.3)\}$ is a formal norm of $p(x, \xi)$ and denote it by $\|p ; \mathbb{M}\|$.

iii) We say that a symbol $p(x, \xi)\left(\in S^{-\infty}\right)$ belongs to a class $\mathscr{R}_{G(\kappa)}$ if for any $\alpha$ there exists a constant $\mathbb{C}_{\alpha}$ such that

$$
\left|p_{(\beta)}^{(\alpha)}(x, \xi)\right| \leqq \mathbb{C}_{\alpha} \mathbb{M}^{-|\beta|} \beta !^{\kappa} \exp \left(-\varepsilon\langle\xi\rangle^{1 / \kappa}\right)
$$

hold with a positive constant $\varepsilon$ independent of $\alpha$ and $\beta$. We call a symbol in $\mathscr{R}_{G(\kappa)}$ a regularizer. We also denote $\inf \left\{C_{\alpha}\right.$ of $\left.(1.4) ;|\alpha| \leqq k\right\}$ by $\|p ; \mathbb{M}\|_{k}$ and call it a formal semi-norm of $p(x, \xi)$.

Remark 1. In the following we call a function $w(\theta)$ in i)-ii) of Definition 1.1 an order function.

Remark 2. When $w(\theta)=\theta^{m}$ for a real $m$ we denote $S_{\rho, \delta, G(\kappa)}[w]$ and $S W F_{1, \delta, G(\kappa)}[w]$ by $S_{\rho, \delta, G(\kappa)}^{m}$ and $S W F_{1, \delta, G(\kappa)}^{m}$.

Remark 3. When $w(\theta)=\exp \left(C \theta^{\sigma}\right)$ for a $\sigma>0$, the classes $S_{\rho, \delta, G(\kappa)}[w]$ and $S W F_{1, \delta, G(\kappa)}[w]$ are symbol classes of exponential type, and these correspond to the classes investigated in [25] and [2].

Remark 4. Formal symbols are investigated in [25] and [16].

Proposition 1.2. Let $w_{j}(\theta), j=1,2$, be order functions such that

$$
w_{j}(\theta) \leqq C_{\varepsilon} \exp \left(\varepsilon \theta^{1 / \kappa}\right) \quad \text { for any } \varepsilon>0 \quad(j=1,2)
$$

and let $P_{j}=p_{j}\left(X, D_{x}\right)$ be pseudo-differential operators with symbols in $S_{\rho, \delta, G(\kappa)}\left[w_{j}\right]$. Then, choosing an order function $w(\theta)$ satisfying $w(\theta) \geqq w_{1}(2 \theta) w_{2}(\theta)$ there exist symbols $q(x, \xi)$ in $S_{\rho, \delta, G(\kappa)}[w]$ and $r(x, \xi)$ in $\mathscr{R}_{G(\kappa)}$ such that the product $\mathbb{P}_{1} \mathbb{P}_{2}$ can be written in the form

$$
P_{1} \mathbb{P}_{2}=q\left(X, D_{x}\right)+r\left(X, D_{x}\right)
$$

Remark. In the above proposition we say that the symbol $q(x, \xi)$ is a main symbol of $P_{1} \mathbb{P}_{2}$ and denote it by $\sigma_{M}\left(P_{1} P_{2}\right)$.

Proof. Write the symbol $\sigma\left(\mathbb{P}_{1} \mathbb{P}_{2}\right)$ as

$$
\begin{aligned}
\sigma\left(P_{1} P_{2}\right)(x, \xi) & =O_{s}-\iint e^{-i y \cdot \eta} p_{1}(x, \xi+\eta) p_{2}(x+y, \xi) d x d \eta \\
& =O_{s}-\iint e^{-i y \cdot \eta}\left(L_{1}^{t}\right)^{n+1} p_{1}(x, \xi+\eta) p_{2}(x+y, \xi) d y d \eta
\end{aligned}
$$

where $d \eta=(2 \pi)^{-n} d \eta$ and $\mathbb{L}_{1}{ }^{t}$ is the transposed operator of $\mathbb{L}_{1}=$ $\left(1+\langle\xi+\eta\rangle^{2 \delta}|y|^{2}\right)^{-1}\left(1+i\langle\xi+\eta\rangle^{2 \delta} y \cdot \nabla_{\eta}\right)$. Denote $\chi(\xi)$ a function in $\gamma^{(\kappa)}$ satis- 
fying

$$
0 \leqq \chi \leqq 1, \quad \chi=1 \quad(|\xi| \leqq 2 / 5), \quad \chi=0 \quad(|\xi| \geqq 1 / 2)
$$

and divide (1.7) as

$$
\begin{aligned}
& \sigma\left(P_{1} P_{2}\right)(x, \xi)=q(x, \xi)+r(x, \xi), \\
& q(x, \xi)=O_{s^{-}} \iint e^{-i y \cdot \eta}\left(L_{1}{ }^{t}\right)^{n+1} p_{1}(x, \xi+\eta) \chi(\eta /\langle\xi\rangle) \\
& \times p_{2}(x+y, \xi) d y d \eta \\
& r(x, \xi)=O_{s^{-}} \iint e^{-i y \cdot \eta}\left(L_{1}^{t}\right)^{n+1} p_{1}(x, \xi+\eta)(1-\chi(\eta /\langle\xi\rangle)) \\
& \times p_{2}(x+y, \xi) d y d \eta .
\end{aligned}
$$

Then, it is easy to prove $q \in S_{\rho, \delta, G(\kappa)}[w]$. Next, we write $r(x, \xi)$ as

$$
\begin{aligned}
r(x, \xi)= & \iint_{|\eta| \leqq \tilde{c}} e^{-i y \cdot \eta}\left(\tilde{L}^{t}\right)^{l_{0}}\left(L_{1}^{t}\right)^{n+1} p_{1}(x, \xi+\eta) \\
& \times(1-\chi(\eta /\langle\xi\rangle)) p_{2}(x+y, \xi) d y d \eta \\
& +\sum_{N=1}^{\infty} \iint_{\tilde{c} N^{\kappa} \leqq|\eta| \leqq \tilde{c}(N+1)^{\kappa}} e^{-i y \cdot \eta}\left(\tilde{L}^{t}\right)^{l_{0}}\left(L_{1}^{t}\right)^{n+1}\left\{p_{1}(x, \xi)(1-\chi(\eta /\langle\xi\rangle))\right. \\
& \left.\times\left(-i|\eta|^{-2} \eta \cdot \nabla_{y}\right)^{N} p_{2}(x+y, \xi)\right\} d y d \eta,
\end{aligned}
$$

where $\tilde{L}=\left(1+\langle\xi\rangle^{2 \delta}|\eta|^{2}\right)^{-1}\left(1-\langle\xi\rangle^{2 \delta} \Delta_{y}\right)$ and $l_{0}=[n /(2(1-\delta))]+1$. Then, using (1.5) we obtain $r \in \mathscr{R}_{G(\kappa)}$ if we take $\tilde{c}$ sufficiently large. Q.E.D.

Remark. In (1.7) the integral is an oscillatory integral, which can be defined as in Section 6 of Chap. 1 in [12].

In order to investigate the product of pseudo-differential operators in $S W F_{1, \delta, G(\kappa)}[w]$ we prepare

Lemma 1.3. Let $w(\theta)$ be an order function and let $\sum p_{j}(x, \xi)$ be a formal symbol satisfying (1.2) with a constant $c(\geqq 1)$. Then, there exists a symbol $p(x, \xi)$ in $S W F_{1, \delta, G(\kappa)}[w]$ such that we have $(1.3)$ for any $\mathbb{N}$.

Proof. We follow [6]. Let $\left\{\psi_{j}(\xi)\right\}$ be a sequence of functions satisfying for a parameter $R$

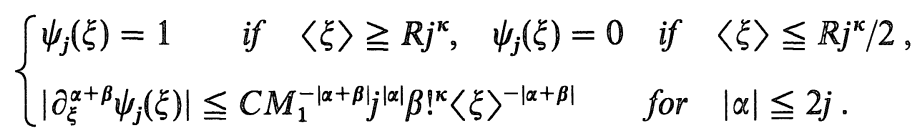

Here, constants $C$ and $M_{1}$ are independent of $j$ and $R$. Define 


$$
p(x, \xi)=\sum_{j=0}^{\infty} p_{j}(x, \xi) \psi_{j}(\xi)(1-\chi(\xi /(3 c)))
$$

for a fixed large constant $R$ and a function $\chi(\xi)$ in $\gamma^{(\kappa)}$ satisfying (1.8). Then, as in [6] we can prove

$$
\begin{gathered}
\left|p_{(\beta)}^{(\alpha)}(x, \xi)\right| \leqq C M^{-|\alpha+\beta|} \alpha !\left(\beta !^{\kappa}+\beta !^{\kappa(1-\delta)}\langle\xi\rangle^{\delta|\beta|}\right)\langle\xi\rangle^{-|\alpha|} w(\langle\xi\rangle) \\
\text { for }\langle\xi\rangle \geqq R|\alpha|^{\kappa}
\end{gathered}
$$

and (1.3). So, by (1.9) an inequality (1.1) holds for $p_{(\beta)}^{(\alpha)}$ when $\langle\xi\rangle \geqq R|\alpha|^{\kappa}$ and it remains to prove (1.1) for $\langle\xi\rangle \leqq \mathbb{R}|\alpha|^{\kappa}$ in order to prove $p(x, \xi) \in S_{1, \delta, G(\kappa)}[w]$. Note

$$
j \leqq(2\langle\xi\rangle / R)^{1 / \kappa} \leqq 2^{1 / \kappa}|\alpha| \quad \text { on } \operatorname{supp} \psi_{j}
$$

when $\langle\xi\rangle \leqq R|\alpha|^{\kappa}$. Then, we can write $p(x, \xi)$ in the form

$$
p(x, \xi)=\sum_{j=0}^{2|\alpha|} p_{j}(x, \xi) \psi_{j}(\xi)(1-\chi(\xi /(3 c))) \quad \text { for }\langle\xi\rangle \leqq R|\alpha|^{\kappa}
$$

and obtain the estimate $(1.1)$ for $p_{(\beta)}^{(\alpha)}(x, \xi)$ in $\langle\xi\rangle \leqq R|\alpha|^{\kappa}$. This proves the lemma.

Q.E.D.

Proposition 1.4. Let $p_{j}(x, \xi)$ be symbols in $S W F_{1, \delta, G(\kappa)}\left[w_{j}\right](j=1,2)$ with $w_{j}(\theta)$ satisfying (1.5). Then, taking an order function $w(\theta)$ satisfying $w(\theta) \geqq$ $w_{1}(\theta) w_{2}(\theta)$, there exist symbols $q(x, \xi)$ in $S W F_{1, \delta, G(\kappa)}[w]$ and $r(x, \xi)$ in $\mathscr{R}_{G(\kappa)}$ such that (1.6) holds and we have for any $N$

$$
\begin{aligned}
\mid \partial_{\xi}^{\alpha} D_{x}^{\beta}( & \left.q(x, \xi)-\sum_{|\gamma|<N} \frac{1}{\gamma !} p_{1}^{(\gamma)}(x, \xi) p_{2(\gamma)}(x, \xi)\right) \mid \\
\leqq & C M^{-(|\alpha+\beta|+N)} \alpha !\left((|\beta|+N) !^{\kappa}+(|\beta|+N) !^{\kappa(1-\delta)}\langle\xi\rangle^{\delta(|\beta|+N)}\right) \\
& \times\langle\xi\rangle^{-N-|\alpha|} w(\langle\xi\rangle) \quad \text { for } \quad|\xi| \geqq c(|\alpha|+N)^{\kappa} .
\end{aligned}
$$

Proof. Let $\sum p_{1, j}(x, \xi)$ and $\sum p_{2, j}(x, \xi)$ be formal symbols associated to $p_{1}(x, \xi)$ and $p_{2}(x, \xi)$, respectively. Define

$$
q_{j}(x, \xi)=\sum_{j^{\prime}+j^{\prime \prime}+|\gamma|=j} \frac{1}{\gamma !} p_{1, j^{\prime}}^{(\gamma)}(x, \xi) p_{2, j^{\prime \prime}(\gamma)}(x, \xi) .
$$

Then, $q_{j}(x, \xi)$ satisfies (1.2) for an order function $w(\theta)$ satisfying $w(\theta) \geqq$ $w_{1}(\theta) w_{2}(\theta)$. Hence, from Lemma 1.3 there exists a symbol $q(x, \xi)$ in $S W F_{1, \delta, G(\kappa)}[w]$ with a formal symbol $\sum q_{j}(x, \xi)$ and $q(x, \xi)$ satisfies (1.10). Now, define

$$
r(x, \xi)=O_{s^{-}} \iint e^{-i y \cdot \eta} p_{1}(x, \xi+\eta) p_{2}(x+y, \xi) d y d \eta-q(x, \xi)
$$


Then the equality (1.6) holds. To prove $r \in \mathscr{R}_{G(\kappa)}$ we write $r(x, \xi)$ as

$$
\begin{aligned}
r(x, \xi)= & \left\{O_{s^{-}} \iint e^{-i y \cdot \eta} p_{1}(x, \xi+\eta) \chi(\eta /\langle\xi\rangle) p_{2}(x+y, \xi) d y d \eta-q(x, \xi)\right\} \\
& \quad+O_{s^{-}} \iint e^{-i y \cdot \eta} p_{1}(x, \xi+\eta)\left(1-\chi(\eta /\langle\xi\rangle) p_{2}(x+y, \xi) d y d \eta\right. \\
\equiv & r_{1}(x, \xi)+r_{2}(x, \xi) .
\end{aligned}
$$

Then, as in the proof of Proposition 1.2 it easily follows $r_{2} \in \mathscr{R}_{G(\kappa)}$. For the proof of $r_{1} \in \mathscr{R}_{G(\kappa)}$, we fix a multi-index $\alpha$ and write $r_{1}^{(\alpha)}(x, \xi)$ as

$$
\begin{aligned}
& r_{1}^{(\alpha)}(x, \xi)= \partial_{\xi}^{\alpha}\left\{\sum_{|\gamma|<N} \frac{1}{\gamma !} p_{1}^{(\gamma)}(x, \xi) p_{2(\gamma)}(x, \xi)-q(x, \xi)\right\} \\
&+ \sum_{|\gamma|<N} \sum_{\left|\gamma^{\prime}\right|=1} \frac{1}{\gamma !} \partial_{\xi}^{\alpha}\left\{\int _ { 0 } ^ { 1 } ( 1 - \theta ) ^ { | \gamma | } \left\{O_{s}-\iint e^{-i y \cdot \eta}\right.\right. \\
& \times p_{1}^{(\gamma)}(x, \xi+\eta) \chi^{\left(\gamma^{\prime}\right)}(\eta /\langle\xi\rangle)\langle\xi\rangle^{-1} \\
&\left.\left.\times p_{2\left(\gamma+\gamma^{\prime}\right)}(x+\theta y, \xi) d y d \eta\right\} d \theta\right\} \\
&+ N \sum_{|\gamma|=N} \partial_{\xi}^{\alpha}\left\{\frac { 1 } { \gamma ! } \int _ { 0 } ^ { 1 } ( 1 - \theta ) ^ { N - 1 } \left\{O_{s^{-}} \iint e^{-i y \cdot \eta} p_{1}^{(\gamma)}(x, \xi+\eta)\right.\right. \\
&\left.\left.\times \chi(\eta /\langle\xi\rangle) p_{2(\gamma)}(x+\theta y, \xi) d y d \eta\right\} d \theta\right\} \\
& \text { (cf. (6.16) of [22]) }
\end{aligned}
$$

Then, for a small constant $\varepsilon>0$ we can prove from (1.10) that, an inequality

$$
\left|r_{1(\beta)}^{(\alpha)}(x, \xi)\right| \leqq C_{\alpha}\left(\beta !^{\kappa}+\beta !^{\kappa(1-\delta)}\langle\xi\rangle^{\delta|\beta|}\right) \exp \left(-\varepsilon\langle\xi\rangle^{1 / \kappa}\right)
$$

holds for $\xi$ satisfying $\mathbb{C}_{1}(N+|\alpha|)^{\kappa} \leqq\langle\xi\rangle \leqq \mathbb{C}_{1}(N+1+|\alpha|)^{\kappa}(N=0,1, \ldots)$ if we take a constant $C_{1}$ large enough. Since $r_{1(\beta)}^{(\alpha)}(x, \xi)$ satisfies $(1.13)$ for $\langle\xi\rangle \leqq C_{1}|\alpha|^{\kappa}$ from (1.11), we have proved that $r_{1}(x, \xi)$ belongs to $\mathscr{R}_{G(\kappa)}$.

Q.E.D.

Remark. In the second term in the right hand side of (1.12) only the terms with $\left|\gamma^{\prime}\right|=1$ appear, and this enables us to obtain (1.13) from (1.12).

Now, we turn to the multi-product of pseudo-differential operators.

Proposition 1.5. Let $p_{j}(x, \xi) \in \mathbb{S}_{\rho, \delta, G(\kappa)}\left[w_{j}\right], j=1,2, \ldots$, and satisfy (1.1) with constant $\mathbb{C}$ and $M$ independent of $j$. Assume that for any $v$

$$
\prod_{j=1}^{v} w_{j}(\theta) \leqq \mathbb{W}_{v, \varepsilon} \exp \left(\varepsilon \theta^{1 / \kappa}\right) \quad \text { for any } \quad \varepsilon>0
$$


Then, the multi-product $Q_{v+1}=P_{1} P_{2} \ldots P_{v+1}$ of pseudo-differential operators $P_{j}=p_{j}\left(X, D_{x}\right)$ has the form

$$
Q_{v+1}=q_{v+1}\left(X, D_{x}\right)+r_{v+1}\left(X, D_{x}\right)
$$

and $q_{v+1}(x, \xi)$ and $r_{v+1}(x, \xi)$ satisfy

$$
\begin{aligned}
\left|q_{v+1(\beta)}^{(\alpha)}(x, \xi)\right| \leqq & A^{v} C^{v+1} M_{1}^{-|\alpha+\beta|}\left(\alpha !^{\kappa}+\alpha !^{\kappa \rho}\langle\xi\rangle^{(1-\rho)|\alpha|}\right) \\
& \times\left(\beta !^{\kappa}+\beta !^{\kappa(1-\delta)}\langle\xi\rangle^{\delta|\beta|}\right)\langle\xi\rangle^{-|\alpha|} \tilde{w}_{v+1}(\langle\xi\rangle)
\end{aligned}
$$

with an order function $\tilde{w}_{v+1}(\theta)$ satisfying $\tilde{w}_{v+1}(\theta) \geqq \prod_{j=1}^{v+1} w_{j}(2 \theta)$ and

$$
\begin{aligned}
\left|r_{v+1(\beta)}^{(\alpha)}(x, \xi)\right| \leqq & A^{v} C^{v+1} C_{\alpha} \tilde{W}_{v+1, \varepsilon} M_{1}^{-|\beta|} \\
& \times\left(\beta !^{\kappa}+\beta !^{\kappa(1-\delta)}\langle\xi\rangle^{\delta|\beta|}\right) \exp \left(-\varepsilon\langle\xi\rangle^{1 / \kappa}\right)
\end{aligned}
$$

for a positive constant $\varepsilon$. Here,

$$
\tilde{W}_{\nu+1, \varepsilon}=\sup _{\theta}\left\{\left(\prod_{j=1}^{v+1} w_{j}(\theta)\right) \exp \left(-\varepsilon \theta^{1 / \kappa}\right)\right\},
$$

and $A$ and $M_{1}$ are constants determined only by the dimension $n$ and $M$ and the constants $C_{\alpha}$ are determined only by $n$ and $\alpha$. All the constants $A, M_{1}$ and $C_{\alpha}$ are independent of $v$.

Proof. For $j$ with $1 \leqq j \leqq v$ we write

$$
p_{j}^{\prime}\left(x, \xi, x^{\prime}\right)=\left(L^{t}\right)^{[n / 2]+1} p_{j}(x, \xi),
$$

with $L=\left(1+\langle\xi\rangle^{2 \delta}\left|x-x^{\prime}\right|^{2}\right)^{-2}\left(1-\langle\xi\rangle^{2 \delta} \Delta_{\xi}\right)$. Then, the symbol $\sigma\left(Q_{v+1}\right)$ of the multi-product $Q_{v+1}$ is written as

$$
\begin{aligned}
\sigma\left(Q_{v+1}\right)=O_{s^{-}} \iint e^{-i \psi} & \prod_{j=1}^{v} p_{j}^{\prime}\left(x+y^{j-1}, \xi+\eta^{j}, x+y^{j}\right) \\
& \times p_{v+1}\left(x+y^{v}, \xi\right) d \tilde{y}^{v} d \tilde{\eta}^{v} \quad\left(y^{0}=0\right),
\end{aligned}
$$

where

$$
\psi=\sum_{j=1}^{v} y^{j} \cdot\left(\eta^{j}-\eta^{j+1}\right) \quad\left(\eta^{v+1}=0\right)
$$

and $d \tilde{y}^{v} d \tilde{\eta}^{v}=d y^{1} \ldots d y^{v} d \eta^{1} \ldots d \eta^{v}$. Take an order function $w_{v+1}^{\prime}(\theta)$ satisfying $w_{v+1}^{\prime}(\theta) \geqq \prod_{j=1}^{v+1} w_{j}(\theta)$. Then, the product $\prod_{j=1}^{v} p_{j}^{\prime}\left(x^{j-1}, \xi^{j}, x^{j+1}\right) p_{v+1}\left(x^{v}, \xi^{v+1}\right)\left(x^{0}=x\right)$ satisfies $(1.20)$ below with $w_{v+1}(\theta)$ replaced by $w_{v+1}^{\prime}(\theta)$. Hence, the proof of Proposition 1.5 is reduced to the following lemma. 
Lemma 1.6. Let $w_{v+1}(\theta)$ be an order function satisfying

$$
w_{v+1}(\theta) \leqq W_{v+1, \varepsilon} \exp \left(\varepsilon \theta^{1 / \kappa}\right) \quad \text { for any } \varepsilon>0
$$

and let $\tilde{p}_{v+1}\left(x, \tilde{\xi}^{v}, \tilde{x}^{v}, \xi^{v+1}\right)=\tilde{p}_{v+1}\left(x, \xi^{1}, x^{1}, \xi^{2}, \ldots, x^{v}, \xi^{v+1}\right)$ be a multiple symbol satisfying

$$
\begin{aligned}
& \left|\partial_{\xi^{1}}^{\alpha^{1}} \partial_{\xi^{2}}^{\alpha^{2}} \ldots \partial_{\xi^{v+1}}^{\alpha^{v+1}} \partial_{x}^{\beta} \partial_{x^{1}}^{\beta^{1}} \cdots \partial_{x^{v}}^{\beta^{v}} \tilde{p}_{v+1}\left(x, \tilde{\xi}^{v}, \tilde{x}^{v}, \xi^{v+1}\right)\right| \\
& \leqq C M^{-\left(\left|\tilde{\alpha}^{v+1}\right|+|\beta|+|\tilde{\beta} v|\right)} \prod_{j=1}^{v+1}\left(\alpha^{j ! \kappa}+\alpha^{j ! \kappa \rho}\left\langle\xi^{j}\right\rangle^{(1-\rho)\left|\alpha^{J}\right|}\right) \\
& \times\left(\beta !^{\kappa}+\beta !^{\kappa(1-\delta)}\left\langle\xi^{1}\right\rangle^{\delta|\beta|}\right) \\
& \times \prod_{j=1}^{v}\left(\beta^{j !^{\kappa}}+\beta^{j ! \kappa(1-\delta)}\left(\left\langle\xi^{j}\right\rangle+\left\langle\xi^{j+1}\right\rangle\right)^{\delta|\beta \jmath|}\right) \\
& \times \prod_{j=1}^{v}\left(1+\left\langle\xi^{j}\right\rangle^{\delta}\left|x^{j-1}-x^{j}\right|\right)^{-(n+1)} \\
& \times\left\{\prod_{j=1}^{v+1}\left\langle\xi^{j}\right\rangle^{-\left|\alpha^{j}\right|}\right\} w_{v+1}\left(\max _{j}\left\langle\xi^{j}\right\rangle\right) \quad\left(x^{0}=x\right),
\end{aligned}
$$

where $\left|\tilde{\alpha}^{v+1}\right|=\left|\alpha^{1}\right|+\cdots+\left|\alpha^{v+1}\right|$ for $\tilde{\alpha}^{v+1}=\left(\alpha^{1}, \ldots, \alpha^{v+1}\right)$ and $\left|\tilde{\beta}^{v}\right|=\left|\beta^{1}\right|+\cdots+$ $\left|\beta^{v}\right|$ for $\tilde{\beta}^{v}=\left(\beta^{1}, \ldots, \beta^{v}\right)$.

Then, the simplified symbol $p_{v+1}(x, \xi)$ defined by

$$
p_{v+1}(x, \xi)=O_{s^{-}} \iint e^{-i \psi} \tilde{p}_{v+1}\left(x, \xi+\eta^{1}, x+y^{1}, \ldots, \xi+\eta^{v}, x+y^{v}, \xi\right) d \tilde{y}^{v} d \tilde{\eta}^{v}
$$

with $\psi$ in (1.18) can be written in the form

$$
p_{v+1}(x, \xi)=q_{v+1}(x, \xi)+r_{v+1}(x, \xi)
$$

and $q_{v+1}(x, \xi)$ and $r_{v+1}(x, \xi)$ have the same estimates (1.16)-(1.17) in Proposition 1.5 with $\tilde{w}_{v+1}(\theta)=w_{v+1}(2 \theta)$ and

$$
\tilde{W}_{v+1, \varepsilon}=\sup _{\theta}\left\{w_{v+1}(\theta) \exp \left(-\varepsilon \theta^{1 / \kappa}\right)\right\} .
$$

Proof. Following [10] we write

$$
\begin{aligned}
p_{v+1}(x, \xi)= & q_{v+1}(x, \xi)+r_{v+1}(x, \xi) \\
q_{v+1}(x, \xi)= & O_{s^{-}} \iint e^{-i \psi} \prod_{j=1}^{v} \chi\left(\eta^{j} /\langle\xi\rangle\right) \\
& \times \tilde{p}_{v+1}\left(x, \xi+\eta^{1}, x+y^{1}, \ldots, \xi+\eta^{v}, x+y^{v}, \xi\right) d \tilde{y}^{v} d \tilde{\eta}^{v}
\end{aligned}
$$




$$
\begin{aligned}
r_{v+1}(x, \xi)= & O_{s^{-}} \iint e^{-i \psi}\left(1-\prod_{j^{\prime}=1}^{v} \chi\left(\eta^{j^{\prime}}\langle\langle\xi\rangle)\right)\right. \\
& \times \tilde{p}_{v+1}\left(x, \xi+\eta^{1}, x+y^{1}, \ldots, \xi+\eta^{v}, x+y^{v}, \xi\right) d \tilde{y}^{v} d \tilde{\eta}^{v} .
\end{aligned}
$$

Setting $\Omega_{0}(j)=\left\{\left(\eta^{1}, \ldots, \eta^{v}\right) ; \quad\left|\eta^{j}\right|=\max _{1 \leqq j^{\prime} \leqq \nu}\left|\eta^{j^{\prime}}\right|>2\langle\xi\rangle / 5, \quad\left|\eta^{j^{\prime}}\right|<\left|\eta^{j}\right| \quad\left(j^{\prime}<j\right)\right.$, $\left.\left|\eta^{j}\right| \leqq c\right\} \quad$ and $\quad \Omega_{N}(j)=\left\{\left(\eta^{1}, \ldots, \eta^{v}\right) ; \quad\left|\eta^{j}\right|=\max _{1 \leqq j^{\prime} \leqq \nu}\left|\eta^{j^{\prime}}\right|>2\langle\xi\rangle / 5, \quad\left|\eta^{j^{\prime}}\right|<\left|\eta^{j}\right|\right.$ $\left.\left(j^{\prime}<j\right), c N^{\kappa} \leqq\left|\eta^{j}\right| \leqq c(N+1)^{\kappa}\right\}(N \geqq 1)$, we rewrite $r_{v+1}(x, \xi)$ as

$$
\begin{aligned}
r_{v+1(\beta)}^{(\alpha)}(x, \xi)= & \sum_{\alpha^{\prime}+\alpha^{\prime \prime}=\alpha} \frac{\alpha !}{\alpha^{\prime} ! \alpha^{\prime \prime} !} O_{s}-\iint e^{-i \psi} \partial_{\xi}^{\alpha^{\prime}}\left(1-\prod_{j^{\prime}=1}^{v} \chi\left(\eta^{j^{\prime}} /\langle\xi\rangle\right)\right) \\
& \times \partial_{\xi}^{\alpha^{\prime \prime}} D_{x}^{\beta} \tilde{p}_{v+1}\left(x, \xi+\eta^{1}, x+y^{1}, \ldots, \xi+\eta^{v}, x+y^{v}, \xi\right) d \tilde{y}^{v} d \tilde{\eta}^{v} \\
= & \sum_{j=1}^{v} \sum_{N=0}^{\infty} \sum_{\alpha^{\prime}+\alpha^{\prime \prime}=\alpha} \frac{\alpha !}{\alpha^{\prime} ! \alpha^{\prime \prime} !} \iint_{R_{\tilde{y} v}^{n v} \times \Omega_{N}(j)} e^{-i \psi} \partial_{\xi}^{\alpha^{\prime}}\left(1-\prod_{j^{\prime}=1}^{v} \chi\left(\eta^{j^{\prime}} /\langle\xi\rangle\right)\right) \\
& \times\left\{-i\left|\eta^{j}\right|^{-2} \eta^{j} \cdot\left(\partial_{y^{j}}+\cdots+\partial_{y^{v}}\right)\right\}^{N} \partial_{\xi}^{\alpha^{\prime \prime}} D_{x}^{\beta} \tilde{p}_{v+1}(x, \\
& \left.\xi+\eta^{1}, x+y^{1}, \ldots, \xi+\eta^{v}, x+y^{v}, \xi\right) d \tilde{y}^{v} d \tilde{\eta}^{v} .
\end{aligned}
$$

Then, we have (1.16) and (1.17) by taking a constant $c$ large enough and using Proposition 1.7 of [21] and the fact that an inequality

$$
w_{v+1}\left(\max _{j^{\prime}}\left\langle\xi+\eta^{j^{\prime}}\right\rangle\right) \leqq w_{v+1}\left(3\left|\eta^{j}\right|\right) \leqq W_{v+1, \varepsilon} \exp \left(3^{1 / \kappa} \varepsilon\left|\eta^{j}\right|\right)
$$

holds in $\bigcup_{N} \Omega_{N}(j)$ from (1.21).

Q.E.D.

Proposition 1.7. Let $p_{l} \in S W F_{1, \delta, G(\kappa)}\left[w_{l}\right], l=1,2, \ldots$, with $\left\{w_{l}(\theta)\right\}$ satisfying (1.14) and let $M$ be a constant independent of $l$. Assume that the formal norms $\left\|p_{l} ; M\right\|$ of $p_{l}(x, \xi)$ are independent of $l$. Then, there exists an order function $\tilde{w}_{v+1}(\theta)$ such that

$$
\tilde{w}_{v+1}(\theta) \geqq \prod_{j=1}^{v+1} w_{j}(\theta)
$$

and the symbols $\sigma\left(Q_{v+1}\right)$ of multi-products $Q_{v+1}$ can be written in the form (1.15) with the symbols $q_{v+1}(x, \xi)$ belonging to $S W F_{1, \delta, G(\kappa)}\left[\tilde{w}_{v+1}\right]$ and symbols $r_{v+1}(x, \xi)$ satisfying (1.17). Moreover, there exist formal symbols $\Sigma q_{v+1, j}(x, \xi)$ associated with $q_{v+1}(x, \xi)$ such that

$$
\begin{aligned}
\left|q_{v+1, j(\beta)}^{(\alpha)}(x, \xi)\right| \leqq & A^{v} C^{v+1} M^{-(|\alpha+\beta|+j)} \alpha ! \\
& \times\left((|\beta|+j) !^{\kappa}+(|\beta|+j) !^{\kappa(1-\delta)}\langle\xi\rangle^{\delta(|\beta|+j)}\right) \\
& \times\langle\xi\rangle^{-j-|\alpha|} \tilde{w}_{v+1}(\langle\xi\rangle) \quad \text { for } \quad|\xi| \geqq c
\end{aligned}
$$


and

$$
\begin{aligned}
\mid \partial_{\xi}^{\alpha} D_{x}^{\beta}( & \left.q_{v+1}(x, \xi)-\sum_{j=0}^{N-1} q_{v+1, j}(x, \xi)\right) \mid \\
\leqq & A^{v} C^{v+1} \mathbb{M}^{-(|\alpha+\beta|+N)} \alpha ! \\
& \times\left((|\beta|+N) !^{\kappa}+(|\beta|+N) !^{\kappa(1-\delta)}\langle\xi\rangle^{\delta(|\beta|+N)}\right) \\
& \times\langle\xi\rangle^{-N-|\alpha|} \tilde{w}_{v+1}(\langle\xi\rangle) \quad \text { for } \quad|\xi| \geqq c(|\alpha|+N)^{\kappa}
\end{aligned}
$$

Proof. Define sequences $\left\{q_{v, j}\right\}_{j=0,1,2, \ldots}$ inductively by

$$
\left\{\begin{array}{l}
q_{1, j}(x, \xi)=p_{1, j}(x, \xi), \\
q_{v+1, j}(x, \xi)=\sum_{|\gamma|+j^{\prime}+j^{\prime \prime}=j} \frac{1}{\gamma !} q_{\nu, j^{\prime}}^{(\gamma)}(x, \xi) p_{v+1, j^{\prime \prime}(\gamma)}(x, \xi),
\end{array}\right.
$$

where $\Sigma p_{l, j}(x, \xi)$ are formal symbols associated with $p_{l}(x, \xi)$. Then by the induction on $v$ we can prove

$$
\begin{aligned}
\left|q_{v+1, j(\beta)}^{(\alpha)}(x, \xi)\right| \leqq & A^{v} \mathbb{C}^{v+1} \mathbb{M}_{1}^{-(|\alpha|+|\beta|+2 j)}(|\alpha|+j) ! \beta ! \\
& \times\left((|\beta|+j)^{\kappa-1}+(|\beta|+j)^{\kappa(1-\delta)-1}\langle\xi\rangle^{\delta}\right)^{|\beta|+j} \\
& \times\langle\xi\rangle^{-|\alpha|-j} \tilde{w}_{v+1}(\langle\xi\rangle) \quad \text { for } \quad|\xi| \geqq c .
\end{aligned}
$$

Hence, applying Lemma 1.3 we can find symbols $q_{v+1}(x, \xi)$ satisfying (1.16) and (1.23)-(1.24). Now, write the multi-products $\mathbb{Q}_{v+1}$ as

$$
\begin{aligned}
\mathcal{Q}_{v+1} \equiv & \mathbb{P}_{1} \mathbb{P}_{2} \ldots P_{v+1} \\
= & q_{v+1}\left(X, D_{x}\right) \\
& +\left\{q_{v}\left(X, D_{X}\right) \mathbb{P}_{v+1}-q_{v+1}\left(X, \mathbb{D}_{x}\right)\right\} \\
& +\left\{q_{v-1}\left(X, D_{x}\right) \mathbb{P}_{v}-q_{v}\left(X, D_{x}\right)\right\} \mathbb{P}_{v+1} \\
& +\cdots \cdots \\
& +\left\{q_{2}\left(X, \mathbb{D}_{x}\right) \mathbb{P}_{3}-q_{3}\left(X, \mathbb{D}_{x}\right)\right\} \mathbb{P}_{4} \ldots \mathbb{P}_{v+1} \\
& +\left\{q_{1}\left(X, D_{x}\right) P_{2}-q_{2}\left(X, \mathbb{D}_{x}\right)\right\} \mathbb{P}_{3} \ldots \mathbb{P}_{v+1}
\end{aligned}
$$

Then, it follows from (1.23)-(1.24) that the terms except the first term in the last member of (1.26) satisfy (1.17). This completes the proof.

Q.E.D.

Combining Proposition 1.5 and Proposition 1.7 with discussion in Section 5 of [22] we obtain

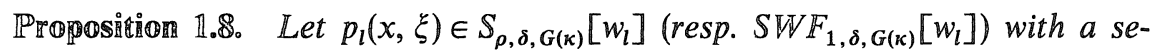
quence $\left\{w_{l}\right\}$ of order functions $w_{l}(\theta)$ satisfying (1.14) and let $\left\{r_{l}^{0}\right\}$ be a sequence 
of regularizers in $\mathscr{R}_{G(\kappa)}$. Assume that for an $M$ the norms $\left\|p_{l} ; M\right\|$ of $p_{l}(x, \xi)$ and the formal semi-norms $\left\|r_{l}^{0} ; M\right\|_{k}$ of $r_{l}^{0}(x, \xi)$ are independent of $l$. Then, the multi-product

$$
Q_{v+1}=\left(P_{1}+R_{1}^{0}\right)\left(P_{2}+R_{2}^{0}\right) \ldots\left(P_{v+1}+R_{v+1}^{0}\right)
$$

of $\mathbb{P}_{l}+R_{l}^{0} \equiv p_{l}\left(X, D_{x}\right)+r_{l}^{0}\left(X, D_{x}\right)$ can be written in the form (1.15) and the symbol $q_{v+1}(x, \xi)$ belongs to $S_{\rho, \delta, G(\kappa)}\left[\tilde{w}_{v+1}\right]$ (resp. $\left.S W F_{1, \delta, G(\kappa)}\left[\tilde{w}_{v+1}\right]\right)$ and satisfies (1.16) (resp. (1.16) and has a formal symbol $\Sigma q_{v+1, j}(x, \xi)$ satisfying (1.23)-(1.24)), and $r_{v+1}(x, \xi)$ satisfies (1.17). Here, $\tilde{w}_{v+1}(\theta)$ is an order function satisfying (1.22).

Finnally we give a result on Neumann series.

Proposition 1.9. Let $p(x, \xi) \in S W F_{1, \delta, G(\kappa)}^{0}$ and assume that its formal norm is sufficiently small. Then, the inverse operator of $I-P$ is represented as $\sum_{v=0}^{\infty} P^{v}$ and there exist symbols $q(x, \xi)$ in $S W F_{1, \delta, G(\kappa)}^{0}$ and $r(x, \xi)$ in $\mathscr{R}_{G(\kappa)}$ such that

$$
\sum_{v=0}^{\infty} P^{v}=q\left(X, D_{x}\right)+r\left(X, D_{x}\right) \quad\left(=(I-P)^{-1}\right)
$$

Proof. For a $(v+1)$-th power $P^{v+1}$ of $P$ we apply Proposition 1.7. Then, $P^{v+1}$ is written as

$$
\mathbb{P}^{v+1}=q_{v+1}\left(X, D_{x}\right)+r_{v+1}\left(X, D_{x}\right)
$$

and $q_{v+1}(x, \xi)$ and $r_{v+1}(x, \xi)$ satisfy $(1.16)-(1.17)$ with $\tilde{w}_{v+1}(\theta)=1$ and $\tilde{W}_{v+1}=1$ and for the formal symbols $\sum q_{v+1, j}(x, \xi)$ we have (1.23)-(1.24). Now, assuming $A\|p ; M\|<1$ for the formal norm $\|p ; M\|$ of $p(x, \xi)$ we define

$$
\begin{gathered}
q(x, \xi)=1+p(x, \xi)+\sum_{v=2}^{\infty} q_{v}(x, \xi), \\
\left\{\begin{array}{l}
q_{0}^{0}(x, \xi)=1+p_{0}(x, \xi)+\sum_{v=2}^{\infty} q_{v, 0}(x, \xi), \\
q_{j}^{0}(x, \xi)=p_{j}(x, \xi)+\sum_{v=2}^{\infty} q_{v, j}(x, \xi) \quad(j \geqq 1)
\end{array}\right.
\end{gathered}
$$

and

$$
r(x, \xi)=\sum_{v=2}^{\infty} r_{v}(x, \xi)
$$

where $\Sigma p_{j}(x, \xi)$ is a formal symbol associated with $p(x, \xi)$. Then, $q(x, \xi)$ and $r(x, \xi)$ are desired symbols and $\Sigma q_{j}^{0}(x, \xi)$ is a formal symbol associated with $q(x, \xi)$.

Q.E.D. 


\section{§2. Calculus of Fourier Integral Operators}

Following [22] we introduce

Definition 2.1. Let $0 \leqq \tau<1$. We say that a phase function $\phi(x, \xi)$ belongs to a class $\mathscr{P}_{G(\kappa)}(\tau)$ if $\phi(x, \xi)$ belongs to a class $\mathscr{P}_{1}(\tau)$ defined in [13] and for $J(x, \xi) \equiv \phi(x, \xi)-x \cdot \xi$ the estimate

$$
\left|J_{(\beta)}^{(\alpha)}(x, \xi)\right| \leqq \tau M^{-(|\alpha|+|\beta|)}(\alpha ! \beta !)^{\kappa}\langle\xi\rangle^{1-|\alpha|}
$$

holds for a constant $M$ independent of $\alpha$ and $\beta$. We also set

$$
\mathscr{P}_{G(\kappa)}=\bigcup_{0 \leqq \tau<1} \mathscr{P}_{G(\kappa)}(\tau) .
$$

For $\phi(x, \xi)$ in $\mathscr{P}_{G(\kappa)}$ and a symbol $p(x, \xi)$ in $S_{\rho, \delta, G(\kappa)}[w]$ we denote by $P_{\phi}=p_{\phi}\left(X, D_{x}\right)$ a Fourier integral operator with the phase function $\phi(x, \xi)$ and the symbol $p(x, \xi)$ and especially we denote by $\mathbb{I}_{\phi}$ the Fourier integral operator with the symbol 1. Moreover, we denote by $\mathbb{I}_{\phi^{*}}$ the conjugate Fourier integral operator with the phase function $\phi(x, \xi)$ and the symbol 1 .

In [22] we have proved

Lemma 2.2 (Proposition 2.5 in [22]). Let $\phi_{j}(x, \xi)$ belong to $\mathscr{P}_{G(\kappa)}\left(\tau_{j}\right), j=1$, 2. Assume $\tau_{1}+\tau_{2}$ is small enough. Then, there exist symbols $p(x, \xi)$ in $\mathbb{S}_{1,0, G(\kappa)}^{0}$ and $r(x, \xi)$ in $\mathscr{R}_{G(\kappa)}$ such that

$$
I_{\phi_{1}} \mathbb{I}_{\phi_{2}}=P_{\Phi}+R
$$

Here, $\Phi(x, \xi)$ is the \#-product $\phi_{1} \# \phi_{2}$ of $\phi_{1}(x, \xi)$ and $\phi_{2}(x, \xi)$, which is defined by

$$
\Phi(x, \xi)=\phi_{1}(x, \Xi)-X \cdot \Xi+\phi_{2}(X, \xi)
$$

with the solution $\{X, \Xi\}(x, \xi)$ of

$$
\left\{\begin{array}{l}
X=\nabla_{\xi} \phi_{1}(x, \Xi), \\
\Xi=\nabla_{x} \phi_{2}(X, \xi) .
\end{array}\right.
$$

Lemma 2.3 (Corolary 2.8 of [22] and Proposition 2.2 of [21]). Let $\phi \in$ $\mathscr{P}_{G(\kappa)}(\tau)$ and assume that $\tau$ is small enough. Then, there exist symbols $p(x, \xi)$ in $S_{1,0, G(\kappa)}^{0}$ and $r(x, \xi)$ in $\mathscr{R}_{G(\kappa)}$ such that

$$
I_{\phi} I_{\phi^{*}}(P+R)=\mathbb{I} \text {. }
$$

For $\rho \geqq 1 / 2$ we denote $S_{\rho, G(\kappa)}[w]=S_{\rho, 1-\rho, G(\kappa)}[w]$. The aim of this section is to prove the following proposition.

Proposition 2.4. Let $\phi_{j}, j=1,2$, be phase functions in $\mathscr{P}_{G(\kappa)}\left(\tau_{j}\right)$ and let $p(x, \xi)$ be a symbol in $S_{\rho, G(\kappa)}[w]$ with $\rho \geqq 1 / 2$ and an order function $w(\theta)$ satis- 
fying

$$
w(\theta) \leqq C_{\varepsilon} \exp \left(\varepsilon \theta^{1 / \kappa}\right) \quad \text { for any } \varepsilon>0 .
$$

Then, there exists a constant $\tau^{0}$ such that if $\tau_{1}+\tau_{2} \leqq \tau^{0}$ we can find symbols $q(x, \xi)$ in $S_{\rho, G(\kappa)}[\tilde{w}]$ for $\tilde{w}(\theta)=w(c \theta)$ with a constant $c(\geqq 1)$ and $r(x, \xi)$ in $\mathscr{R}_{G(\kappa)}$ such that

$$
I_{\phi_{1}} P I_{\phi_{2}}=I_{\phi} Q+R
$$

where $\Phi=\phi_{1} \# \phi_{2}$.

For the proof we prepare two lemmas. Then, combining Proposition 1.2, Lemma 2.2 and Lemma 2.3 we can obtain Proposition 2.4 by regarding discussion in $\S 2$ of [21] (cf. Lemma 2.10).

Lemma 2.5. Let $p(x, \xi) \in S_{\rho, G(\kappa)}[w]$ with $\rho \geqq 1 / 2$ and with an order function $w(\theta)$ satisfying (2.1), and let $\phi(x, \xi) \in \mathscr{P}_{\mathbf{G}(\kappa)}$. Then, there exist symbols $q(x, \xi)$ in $S_{\rho, G(\kappa)}[\tilde{w}]$ with $\tilde{w}(\theta)=w(2 \theta)$ and $r(x, \xi)$ in $\mathscr{R}_{G(\kappa)}$ such that we have

$$
P I_{\phi}=Q_{\phi}+R \text {. }
$$

Moreover, for any $N$ there exists a symbol $q_{N}(x, \xi)$ satisfying $\langle\xi\rangle^{(2 \rho-1) N} q_{N}(x, \xi) \in$ $S_{\rho, G(\kappa)}[\tilde{w}]$ with $\tilde{w}(\theta)=w(2 \theta)$ such that

$$
q(x, \xi)=\sum_{|\gamma|<N} \frac{1}{\gamma !} D_{x^{\prime}}^{\gamma}\left(p^{(\gamma)}\left(x, \tilde{\nabla}_{x} \phi\left(x, x^{\prime} ; \xi\right)\right)\right)_{\mid x^{\prime}=x}+q_{N}(x, \xi)
$$

where $\left.\tilde{\nabla}_{x} \phi\left(x, x^{\prime} ; \xi\right)=\int_{0}^{1} \nabla_{x} \phi\left(x^{\prime}+\theta\left(x-x^{\prime}\right), \xi\right) d \theta\right)$.

Proof (cf. Proposition 2.2 of [22]). From the proof of Theorem 2.2-1) in Chap. 10 of [12], the symbol of $P I_{\phi}$ is written as

$$
\sigma\left(P I_{\phi}\right)=O_{s}-\iint e^{-i y \cdot \eta} p\left(x, \tilde{\nabla}_{x} \phi(x, x+y ; \xi)+\eta\right) d y d \eta .
$$

Using $\chi$ in $\gamma^{(\kappa)}$ satisfying (1.8) we divide (2.3) as

$$
\begin{aligned}
& q(x, \xi)=O_{s^{-}} \iint e^{-i y \cdot \eta} p\left(x, \tilde{\nabla}_{x} \phi(x, x+y ; \xi)+\eta\right) \chi(\eta /\langle\xi\rangle) d y d \eta, \\
& r(x, \xi)=O_{s^{-}} \iint e^{-i y \cdot \eta} p\left(x, \tilde{\nabla}_{x} \phi(x, x+y ; \xi)+\eta\right)(1-\chi(\eta /\langle\xi\rangle)) d y d \eta .
\end{aligned}
$$

Then, the symbols $q(x, \xi)$ and $r(x, \xi)$ are desired symbols when we use (2.1) to prove $r(x, \xi) \in \mathscr{R}_{G(\kappa)}$. For the proof of (2.2) we use the Taylor expansion for $q(x, \xi)$. Then, we have 


$$
\begin{aligned}
q(x, \xi)= & \sum_{|\gamma|<N} \frac{1}{\gamma !} \mathbb{D}_{y}^{\gamma}\left(p^{(\gamma)}\left(x, \tilde{\nabla}_{x} \phi(x, x+y ; \xi)\right)\right)_{\mid y=0}+\sum_{|\gamma|=N} \frac{N}{\gamma !} \int_{0}^{1}(1-\theta)^{N-1} \\
& \times\left\{O _ { s ^ { - } } \int \int e ^ { - i y \cdot \eta } \partial _ { \eta } ^ { \gamma } \mathbb { D } _ { y } ^ { \gamma } \left\{p\left(x, \tilde{\nabla}_{x} \phi(x, x+y, \xi)+\theta \eta\right)\right.\right. \\
& \times \chi(\theta \eta /\langle\xi\rangle)\} d y d \eta\} d \theta
\end{aligned}
$$

and get (2.2).

Q.E.D.

Remark. In the above lemma $Q_{\phi}$ is a Fourier integral operator with infinite order if $w(\theta)$ is an exponential function. We note that Fourier integral operators with infinite order are also considered in [5].

Lemma 2.6. Let $p(x, \xi) \in \mathbb{S}_{\rho, G(\kappa)}[w]$ with $\rho \geqq 1 / 2$ and $w(\theta)$ satisfying (2.1), and let $\phi(x, \xi) \in \mathscr{P}_{G(\kappa)}$. Then, there exist symbols $q(x, \xi)$ in $\mathbb{S}_{\rho, G(\kappa)}[\tilde{w}]$ with $\tilde{w}(\theta)=$ $w(2 \theta)$ and $r(x, \xi)$ in $\mathscr{R}_{G(\kappa)}$ such that we have

$$
\mathbb{I}_{\phi} \cdot \mathbb{P}_{\phi}=Q+\mathbb{R}
$$

Proof. From the proof of Theorem 1.7 in Chap. 10 of [12] we have

$$
\sigma\left(\mathbb{I}_{\phi^{*}} \mathbb{P}_{\phi}\right)=\mathbb{O}_{s^{-}} \iint e^{-i y \cdot \eta} q^{\prime}(\xi+\eta, x+y, \xi) d y d \eta,
$$

for

$$
q^{\prime}\left(\xi, x^{\prime}, \xi^{\prime}\right)=\left\{p\left(z, \xi^{\prime}\right)\left|\operatorname{det} \frac{\partial}{\partial x} \tilde{\nabla}_{\xi} \phi\left(z ; \xi, \xi^{\prime}\right)\right|^{-1}\right\}_{\mid z=\tilde{\nabla}_{\xi} \phi^{-1}\left(x^{\prime} ; \xi, \xi^{\prime}\right)}
$$

where $\tilde{\nabla}_{\xi} \phi\left(x^{\prime} ; \xi, \xi^{\prime}\right)=\int_{0}^{1} \nabla_{\xi} \phi\left(x^{\prime}, \xi^{\prime}+\theta\left(\xi+\xi^{\prime}\right)\right) d \theta$, and $z=\tilde{\nabla}_{\xi} \phi^{-1}\left(x^{\prime} ; \xi, \xi^{\prime}\right)$ is the inverse function of $x^{\prime}=\tilde{\nabla}_{\xi} \phi\left(z ; \xi, \xi^{\prime}\right)$. Now, we write

$$
\begin{aligned}
& q(x, \xi)=O_{s^{-}} \iint e^{-i y \cdot \eta} q^{\prime}(\xi+\eta, x+y, \xi) \chi(\eta /\langle\xi\rangle) d y d \eta, \\
& r(x, \xi)=O_{s^{-}} \iint e^{-i y \cdot \eta} q^{\prime}(\xi+\eta, x+y, \xi)(1-\chi(\eta /\langle\xi\rangle)) d y d \eta,
\end{aligned}
$$

with $\chi \in \gamma^{(\kappa)}$ satisfying (1.8). Then, using Lemma 4.2-ii) in [22] we obtain the lemma.

Q.E.D.

\section{§3. Prelingmi̊mmry}

First, we introduce symbol classes which we use in the following sections. Let $p(\tilde{t}, x, \xi)$ be a symbol with a parameter $\tilde{t}$. In order to simplify the notation 
below, we also denote by $S_{\rho, \delta, G(\kappa)}[w]$ a class of symbols $p(\tilde{t}, x, \xi)$ satisfying the following: $p(\tilde{t}, x, \xi)$ is a continuous function in $(\tilde{t}, x, \xi)$ with all continuous derivatives with respect to $x$ and $\xi$; belongs to $S_{\rho, \delta, G(\kappa)}[w]$ for any fixed $\tilde{t}$ and for an $M$ independent of $\tilde{t}$ the formal norm $\|p(\tilde{t}, \cdot, \cdot) ; M\|$ is bounded in $\tilde{t}$. Similarly we use $S W F_{1, \delta, G(\kappa)}[w]$ and $\mathscr{R}_{G(\kappa)}$ for classes of symbols $p(\tilde{t}, x, \xi)$ depending on a parameter $\tilde{t}$ and $p(\tilde{t}, x, \xi)$ belong to the corresponding symbol classes.

Let $\zeta$ be a parameter not less than 1 and denote

$$
\left\{\begin{array}{l}
\mu(x, \xi ; \zeta)=\left(g(x)^{2 l^{\prime}}\langle\xi\rangle^{2(1-\sigma)}+\zeta^{2}\right)^{1 / 2} \\
h(t, x, \xi ; \zeta)=t+\zeta^{\omega} \mu(x, \xi ; \zeta)^{-\omega}
\end{array}\right.
$$

where $l^{\prime}$ is an integer in $(\mathbb{A}-1), g(x)$ is in $(\mathbb{A}-3), \sigma$ is defined by (4) and $\omega=1 /(l+1)$. In what follows, $\delta$ is always equal to $(1-\sigma) / l^{\prime}$. Following [17] we introduce

Definition 3.1. i) Let $p(t, x, \xi ; \zeta)$ be a symbol with a parameter $t$ and $\zeta$. For real numbers $m, m^{\prime}, m^{\prime \prime}$ and $\rho$ with $\delta \leqq \rho \leqq 1$ we say that $p(t, x, \xi ; \zeta)$ belongs to a class $\tilde{S}_{\rho, \delta, G(\kappa)}\left[m, m^{\prime}, m^{\prime \prime}\right]$ if $p(t, x, \xi ; \zeta) /\left\{\mu(x, \xi ; \zeta)^{m^{\prime}} h(t, x, \xi ; \zeta)^{m^{\prime \prime}}\right\}$ belongs to $S_{\rho, \delta, G(\kappa)}^{m}$ and its formal norm

$$
\left\|p ; M ;\left[m, m^{\prime}, m^{\prime \prime}\right]\right\| \equiv\left\|p(t, \cdot, \cdot \zeta) /\left\{\mu(\cdot, \cdot \zeta)^{m^{\prime}} h(t, \cdot, \cdot \zeta \zeta)^{m^{\prime \prime}}\right\} ; M\right\|
$$

is independent of $t$ and $\zeta$. Moreover, we say that a symbol $p(t, x, \xi ; \zeta)$ in $\tilde{S}_{\rho, \delta, G(\kappa)}\left[m, m^{\prime}, m^{\prime \prime}\right]$ belongs to a class $S_{\rho, \delta, G(\kappa)}\left[m, m^{\prime}, m^{\prime \prime}\right]$ if $p(t, x, \xi ; \zeta)$ is also infinitely differentiable with respect to $t ; \partial_{t}^{\gamma} p(t, x, \xi ; \zeta)$ belongs to $\widetilde{S}_{\rho, \delta, G(\kappa)}\left[m, m^{\prime}\right.$, $\left.m^{\prime \prime}-\gamma\right]$ for any $\gamma$ and there exist constants $C$ and $M$ independent of $\gamma$ such that

$$
\left\|\partial_{t}^{\gamma} p(t, \cdot, \cdot \zeta) ; M ;\left[m, m^{\prime}, m^{\prime \prime}-\gamma\right]\right\| \leqq C M^{-\gamma} \gamma ! .
$$

ii) Let $p(t, x, \xi ; \zeta)$ be a symbol in $S_{1, \delta, G(\kappa)}\left[m, m^{\prime}, m^{\prime \prime}\right]$. We say that $p(t, x, \xi ; \zeta)$ belongs to a class $S_{1, \delta, G(\kappa)}\left[m, m^{\prime}, m^{\prime \prime}\right]$ if $p(t, x, \xi ; \zeta)$ satisfies in addition

$$
\begin{aligned}
\mid \partial_{t}^{\gamma} p_{(\beta)}^{(\alpha)}(t, x, & \xi \zeta) \mid \leqq C M^{-(|\alpha+\beta|+\gamma)} \alpha ! \\
& \times\left(\beta !^{\kappa}+\beta !^{\kappa(1-\delta)}\langle\xi\rangle^{\delta|\beta|}\right) \gamma !\langle\xi\rangle^{m-|\alpha|} \mu(x, \xi ; \zeta)^{m^{\prime}} \\
& \times h(t, x, \xi ; \zeta)^{m^{\prime \prime}-\gamma} \quad \text { for } \quad|\xi| \geqq c
\end{aligned}
$$

for a constant $c>0$.

iii) Let $p(t, x, \xi ; \zeta)$ be a symbol in $S_{1, \delta, G(\kappa)}\left[m, m^{\prime}, m^{\prime \prime}\right]$. We say that a symbol $p(t, x, \xi ; \zeta)$ belongs to a class $S W F_{1, \delta, G(\kappa)}\left[m, m^{\prime}, m^{\prime \prime}\right]$ if $\left(\partial_{t}^{\gamma} p(t, x, \xi\right.$; $\zeta)) /\left\{\mu(x, \xi ; \zeta)^{m^{\prime}} h(t, x, \xi ; \zeta)^{m^{\prime \prime}-\gamma}\right\}$ belongs to $S W F_{1, \delta, G(\kappa)}^{m}$ and for a formal symbol $\sum p_{j}(t, x, \xi ; \zeta), p(t, x, \xi ; \zeta)$ has uniform estimates similar to(1.2)-(1.3) with respect to $t$ and $\zeta$. 
Remark 1. For the symbols $\mu(x, \xi ; \zeta)$ and $h(t, x, \xi ; \zeta)$ in (3.1) we have $\mu(x, \xi ; \zeta) \in \stackrel{\circ}{S}_{1, \delta, G(\kappa)}[0,1,0]$ and $h(t, x, \xi ; \zeta) \in \stackrel{\circ}{1, \delta, G(\kappa)}[0,0,1]$.

Remark 2. For every $p(t, x, \xi ; \zeta) \in \stackrel{S}{1, \delta, G(\kappa)}^{\circ}\left[m, m^{\prime}, m^{\prime \prime}\right]$ we set $p_{0}(t, x, \xi ; \zeta)=$ $p(t, x, \xi ; \zeta)$ and $p_{j}(t, x, \xi ; \zeta)=0$ for $j \geqq 1$. Then, $\sum p_{j}(t, x, \xi ; \zeta)$ is a formal symbol associated with $p(t, x, \xi ; \zeta)$. So, we can regard symbols in $S_{1, \delta, G(\kappa)}^{\circ}\left[m, m^{\prime}, m^{\prime \prime}\right]$ as symbols in $S W F_{1, \delta, G(\kappa)}\left[m, m^{\prime}, m^{\prime \prime}\right]$.

For a symbol class of Hermite operators we introduce

Definition 3.2 (cf. [3]). Let $m$ and $m^{\prime}$ be real numbers. We say that a symbol $p(t, x, \xi)$ belongs to a class $\mathscr{H}_{1, \delta, G(\kappa)}\left[m, m^{\prime}\right]$ if $p(t, x, \xi)$ satisfies

$$
\begin{aligned}
\left|p_{(\beta)}^{(\alpha)}(t, x, \xi)\right| \leqq & C M^{-|\alpha+\beta|} \alpha !^{\kappa}\left(\beta !^{\kappa}+\beta !^{\kappa(1-\delta)}\langle\xi\rangle^{\delta|\beta|}\right) \\
& \times\langle\xi\rangle^{m-|\alpha|} \mu(x, \xi)^{m^{\prime}} \exp \left(-\varepsilon t^{l+1} \mu(x, \xi)\right)
\end{aligned}
$$

for a positive constant $\varepsilon$, where $\mu(x, \xi)=\left(g(x)^{2 l^{\prime}}\langle\xi\rangle^{2(1-\sigma)}+1\right)^{1 / 2}(\equiv \mu(x, \xi ; 1))$.

Remark. In [17] we assumed an estimate for derivatives of symbols $p(t, x, \xi)$ of Hermite operators with respect to $t$. But, in the following we do not need estimates for derivatives of $p(t, x, \xi)$ with respect to $t$.

Lemma 3.3. Let $h(t, x, \xi ; \zeta)$ be a symbol in (3.1). Then, there exists a $\zeta_{1}$ such that for $\zeta \geqq \zeta_{1}$ the operator $h\left(t, X, D_{x} ; \zeta\right)$ has an inverse operator $h\left(t, X, D_{x} ; \zeta\right)^{-1}$ and it has the form

$$
h\left(t, X, D_{x} ; \zeta\right)^{-1}=p\left(t, X, D_{x} ; \zeta\right)+r\left(t, \mathbb{X}, \mathbb{D}_{x} ; \zeta\right)
$$

with symbol $p(t, x, \xi ; \zeta)$ in $S W F_{1, \delta, G(\kappa)}[0,0,-1]$ and $r(t, x, \xi ; \zeta)$ in $\mathscr{R}_{G(\kappa)}$.

Proof. Set $p_{1}(t, x, \xi ; \zeta)=h(t, x, \xi ; \zeta)^{-1}\left(\in S_{1, \delta, G(\kappa)}[0,0,-1]\right)$. Then, by Proposition 1.4 there exist symbols $p_{2}(t, x, \xi ; \zeta)$ in $S W F_{1, \delta, G(\kappa)}\left[\delta-1,-1 / l^{\prime}, 0\right]$ and $r_{1}(t, x, \xi ; \zeta)$ in $\mathscr{R}_{G(\kappa)}$ such that

$$
p_{1}\left(t, X, D_{x} ; \zeta\right) h\left(t, X, D_{x} ; \zeta\right)=I+p_{2}\left(t, X, D_{x} ; \zeta\right)+r_{1}\left(t, X, D_{x} ; \zeta\right)
$$

holds for $\zeta^{-1} r(t, x, \xi ; \zeta)$ is bounded in $\mathscr{R}_{G(\kappa)}$. Consider $p_{2}(t, x, \xi ; \zeta)$ is the symbol in $S W F_{1, \delta, G(\kappa)}[0,0,0]$. Then its formal norm is estimated by

$$
\left\|p_{2}(t, \cdot ; \zeta)\right\| \leqq C \zeta^{-1 / l^{\prime}} .
$$

So, from Proposition 1.9 and discussion in Section 5 of [22], there exists an inverse operator of $I+p_{2}\left(t, X, D_{x} ; \zeta\right)+r_{1}\left(t, X, D_{x} ; \zeta\right)$ with the form

$$
\left(I+p_{2}\left(t, X, D_{x} ; \zeta\right)+r_{1}\left(t, X, D_{x} ; \zeta\right)\right)^{-1}=p_{3}\left(t, X, D_{x} ; \zeta\right)+r_{2}\left(t, X, D_{x} ; \zeta\right)
$$

for $p_{3}(t, x, \xi ; \zeta) \in S W F_{1, \delta, G(\kappa)}[0,0,0]$ and $r_{2}(t, x, \xi ; \zeta) \in \mathscr{R}_{G(\kappa)}$ if $\zeta \geqq \zeta_{1}$ for a large $\zeta_{1}$. Set

$$
H^{-1}=\left(I+p_{2}\left(t, X, D_{x} ; \zeta\right)+r_{1}\left(t, X, D_{x} ; \zeta\right)\right)^{-1} p_{1}\left(t, X, D_{x} ; \zeta\right)
$$


Then, $H^{-1}$ is a left inverse operator of $h\left(t, X, D_{x} ; \zeta\right)$ and it has the form (3.2). It easily follows that $H^{-1}$ is also a right inverse operator and this concludes the proof.

Q.E.D.

For $\chi(\xi)$ in $\gamma^{(\kappa)}$ with $(1.8)$ we define

$$
\lambda_{0}(t, x, \xi)=\left(\sum_{j, j^{\prime}} a_{j, j^{\prime}}(t, x) \xi_{j} \xi_{j^{\prime}}(1-\chi(\xi))+\chi(\xi / 3)\right)^{1 / 2} .
$$

Then, the (modified) characteristic roots of $L$ in (6) are

$$
\lambda_{ \pm}(t, x, \xi)= \pm t^{l} g(x)^{l^{\prime}} \lambda_{0}(t, x, \xi) .
$$

Lemma 3.4. Let $\phi_{ \pm}(t, s ; x, \xi)$ be phase functions corresponding to $\lambda_{ \pm}(t, x, \xi)$. Then, $\phi_{ \pm}(t, s ; x, \xi)$ belong to $\mathscr{P}_{G(k)}(c|t-s|)$ for a constant $c$, and $\phi_{ \pm}(t, s ; x, \xi)-x \cdot \xi$ belong to $S_{1,0, G(\kappa)}^{1}$ and satisfy

$$
\phi_{ \pm}(t, s ; x, \xi)-x \cdot \xi= \pm g(x)^{l^{\prime}} \int_{s}^{t} \theta^{l} \lambda_{0}\left(\theta, x, \nabla_{x} \phi_{ \pm}(\theta, s ; x, \xi)\right) d \theta
$$

This lemma follows from Proposition 3.1 in [22] and Proposition 3.1 in $[15]$.

\section{Lemma 3.5. Define}

$$
\begin{aligned}
\tilde{\lambda}(t, x, \xi ; \zeta)= & \left\{t^{l}+\zeta^{\omega l} \mu(x, \xi ; \zeta)^{-\omega l} \exp \left(-t^{l+1} \mu(x, \xi ; \zeta) / \zeta\right)\right\} \\
& \times\left\{g(x)^{l^{\prime}} \lambda_{0}(t, x, \xi)+i \zeta\langle\xi\rangle^{\sigma} \exp (-\mu(x, \xi ; \zeta) / \zeta)\right\}
\end{aligned}
$$

with $\lambda_{0}(t, x, \xi)$ of (3.3). Then, $\tilde{\lambda}(t, x, \xi ; \zeta)$ belongs to $S_{1, \delta, G(\kappa)}^{\circ}[\sigma, 1, l]$ and

$$
|\tilde{\lambda}(t, x, \xi ; \zeta)| \geqq C h(t, x, \xi ; \zeta)^{l} \mu(x, \xi ; \zeta)\langle\xi\rangle^{\sigma}
$$

holds with a positive constant $C$ independent of $\zeta$. For any fixed $\zeta$ we have

$$
\tilde{\lambda}(t, x, \xi ; \zeta)-t^{l} g(x)^{l^{\prime}} \lambda_{0}(t, x, \xi) \in \mathscr{H}_{1, \delta, G(x)}[\sigma, \omega] .
$$

Proof. Set $I_{1}=t^{l}+\zeta^{\omega l} \mu(x, \xi ; \zeta)^{-\omega l} \exp \left(-t^{l+1} \mu(x, \xi ; \zeta) / \zeta\right) \quad$ and $\quad I_{2}=$ $g(x)^{l^{\prime}} \lambda_{0}(t, x, \xi)+i \zeta\langle\xi\rangle^{\sigma} \exp (-\mu(x, \xi ; \zeta) / \zeta)$. Then, writing $\mu(x, \xi ; \zeta)$ simply by $\mu$, we have

$$
I_{1} \geqq t^{l} \geqq 2^{-l}\left(t+\zeta^{\omega} \mu^{-\omega}\right)^{l}
$$

when $t \geqq \zeta^{\omega} \mu^{-\omega}$ and

$$
I_{1} \geqq\left(\zeta^{\omega} \mu^{-\omega}\right)^{l} e^{-1} \geqq 2^{-l} e^{-1}\left(t+\zeta^{\omega} \mu^{-\omega}\right)^{l}
$$

when $t \leqq \zeta^{\omega} \mu^{-\omega}$, since we have $0 \leqq t \leqq T$. Similarly, we have

$$
\begin{aligned}
\left|I_{2}\right| & \geqq\left(\left|g(x)^{l^{\prime}} \lambda_{0}(t, x, \xi)\right|+\zeta\langle\xi\rangle^{\sigma} \exp (-\mu / \zeta)\right) / \sqrt{2} \\
& \geqq C \mu(x, \xi ; \zeta)\langle\xi\rangle^{\sigma} .
\end{aligned}
$$


Combining these results we have (3.7). For the proof of (3.8) we write

$$
\begin{aligned}
\tilde{\lambda}(t, & x, \xi ; \zeta)-t^{l} g(x)^{l^{\prime}} \lambda_{0}(t, x, \xi) \\
= & \zeta^{\omega l} \mu^{-\omega l} \exp \left(-t^{l+1} \mu(x, \xi ; \zeta) / \zeta\right) \\
& \left.\times g(x)^{l^{\prime}} \lambda_{0}(t, x, \xi)+i \zeta\langle\xi\rangle^{\sigma} \exp (-\mu(x, \xi ; \zeta) / \zeta)\right\} \\
& +i t^{l} \zeta\langle\xi\rangle^{\sigma} \exp (-\mu(x, \xi ; \zeta) / \zeta) .
\end{aligned}
$$

Then, we get (3.8) since we have $\left|t^{l} \zeta\langle\zeta\rangle^{\sigma} \exp (-\mu(x, \xi ; \zeta) / \zeta)\right| \leqq C\langle\xi\rangle^{\sigma} \mu(x, \xi)^{\omega} \times$ $\exp \left(-\varepsilon t^{l+1} \mu(x, \xi)\right)$ with constants $C$ and $\varepsilon$ depending on $\zeta$.

Q.E.D.

Let $\left\{\lambda_{j}(t, x, \xi)\right\}_{j=1}^{\infty}$ be a sequence of $\lambda_{j}(t, x, \xi)=\lambda_{+}(t, x, \xi)$ or $\lambda_{j}(t, x, \xi)=$ $\lambda_{-}(t, x, \xi)$, and let $\phi_{j}(t, s) \equiv \phi_{j}(t, s ; x, \xi)$ be the phase function corresponding to $\lambda_{j}(t, x, \xi)$. Then, using Proposition 2.4 in [21], the equation

$$
\begin{aligned}
& \left\{\begin{array}{l}
X_{v}^{j}=\nabla_{\xi} \phi_{j}\left(t_{j-1}, t_{j} ; \mathbb{X}_{v}^{j-1}, \Xi_{v}^{j}\right), \\
\Xi_{v}^{j}=\nabla_{x} \phi_{j+1}\left(t_{j}, t_{j+1} ; \mathbb{X}_{v}^{j}, \Xi_{v}^{j+1}\right), \quad j=1, \ldots, v
\end{array}\right. \\
& \left(X_{v}^{0}=x, \Xi_{v}^{v+1}=\xi ; t_{0}=t, t_{v+1}=s\right)
\end{aligned}
$$

has a solution $\left\{\mathbb{X}_{v}^{j}, \Xi_{v}^{j}\right\}_{j=1}^{v}=\left\{\mathbb{X}_{v}^{j}, \Xi_{v}^{j}\right\}_{j=1}^{v}\left(t, \tilde{t}^{v}, s ; x, \xi\right)$ for $\quad \tilde{t}^{v}=\left(t_{1}, \ldots, t_{v}\right)$ satisfying

$$
0 \leqq s \leqq t_{v} \leqq \cdots \leqq t_{1} \leqq t \leqq \mathbb{T}_{1}
$$

if $T_{1}$ is sufficiently small. Hence, a multi-\#-product $\Phi_{v+1} \equiv \Phi_{v+1}\left(t, \tilde{t}^{v}, s ; x, \xi\right)=$ $\left(\phi_{1}\left(t, t_{1}\right) \# \phi_{2}\left(t_{1}, t_{2}\right) \# \cdots \# \phi_{v+1}\left(t_{v}, s\right)\right)(x, \xi)$ of $\phi_{j}\left(t_{j-1}, t_{j} ; x, \xi\right), j=1, \ldots, v+1$, is defined by

$$
\begin{gathered}
\Phi_{v+1}=\sum_{j=1}^{v}\left(\phi_{j}\left(t_{j-1}, t_{j} ; \mathbb{X}_{v}^{j-1}, \Xi_{v}^{j}\right)-\mathbb{X}_{v}^{j} \cdot \Xi_{v}^{j}\right)+\phi_{v+1}\left(t_{v}, s ; X_{v}^{v}, \xi\right) \\
\left(X_{v}^{0}=x\right) .
\end{gathered}
$$

Lemma 3.6. Let $\left\{X_{v}^{j}, \Xi_{v}^{j}\right\}_{j=1}^{v}=\left\{X_{v}^{j}, \Xi_{v}^{j}\right\}_{j=1}^{v}\left(t, \tilde{t}^{v}, s ; x, \xi\right)$ be a solution of (3.9). Then, if $T_{1}$ is small enough, we can find a positive constant $\mathbb{C}$ such that

$$
\mathbb{C}^{-1}|g(x)| \leqq\left|g\left(\mathbb{X}_{v}^{j}\right)\right| \leqq \mathbb{C}|g(x)| \quad(j=1, \ldots, v)
$$

hold for $\tilde{t}^{v}$ satisfying (3.10).

Proof. From (3.9) and (3.5) we have

$$
\begin{aligned}
X_{v}^{j}-x & =\sum_{m=1}^{j}\left(X_{v}^{m}-X_{v}^{m-1}\right) \\
& =\sum_{m=1}^{j}\left(\nabla_{\xi} \phi_{m}\left(t_{m-1}, t_{m} ; X_{v}^{m-1}, \Xi_{v}^{m}\right)-X_{v}^{m-1}\right) \\
& =\sum_{m=1}^{j} g\left(\mathbb{X}_{v}^{m-1}\right)^{l^{\prime}} \int_{t_{m}}^{t_{m-1}} \theta^{l} \nabla_{\xi}\left(\lambda_{m}^{0}\left(\theta, x, \nabla_{x} \phi_{m}\left(\theta, t_{m} ; X_{v}^{m-1}, \Xi_{v}^{m}\right)\right)\right) d \theta
\end{aligned}
$$


where $\lambda_{m}^{0}(t, x, \xi)= \pm \lambda_{0}(t, x, \xi) \quad$ when $\quad \lambda_{m}(t, x, \xi)= \pm t^{l} g(x)^{l^{\prime}} \lambda_{0}(t, x, \xi)$. Hence, setting

$$
G=\max \left\{|g(x)|,\left|g\left(X_{v}^{j}\right)\right| \quad(j=1, \ldots, v)\right\}
$$

we have

$$
\begin{aligned}
|| g\left(X_{v}^{j}\right)|-| g(x)|| & \leqq\left|g\left(X_{v}^{j}\right)-g(x)\right| \leqq C\left|X_{v}^{j}-x\right| \\
& \leqq C^{\prime} \sum_{m=1}^{j}\left|g\left(X_{v}^{m}\right)\right|\left(t_{m-1}-t_{m}\right) \\
& \leqq C^{\prime} T_{1} G .
\end{aligned}
$$

Consequently, if $T_{1}$ satisfies $C^{\prime} T_{1} \leqq 1 / 3$ we have

$$
\frac{1}{2} G \leqq|g(x)| \leqq 2 G \quad(j=0, \ldots, v)
$$

and (3.12).

Q.E.D.

Lemma 3.7. Assume $\sigma^{\prime}$ satisfies (5). Then, for any positive constant $\varepsilon$ there exists a constant $M \equiv M_{\varepsilon}$ such that the multi-\#-product $\Phi_{v+1}$ of (3.11) satisfies

$$
\begin{aligned}
& \left|\partial_{\xi}^{\alpha} \partial_{x}^{\beta} \exp \left[i\left(\Phi_{v+1}-x \cdot \xi\right)\right]\right| \\
& \quad \leqq C M^{-|\alpha+\beta|} \alpha !^{\kappa} \beta !^{\kappa}\langle\xi\rangle^{-|\alpha|} \exp \left[\varepsilon t^{l+1} \mu(x, \xi)+\langle\xi\rangle^{\sigma^{\prime}}\right]
\end{aligned}
$$

for $\left(t, \tilde{t}^{\nu}, s\right)$ satisfying (3.10), where $T_{1}$ is the constant in Lemma 3.6.

Remark. We note that we can take the $\sigma^{\prime}$ satisfying (5) since we have $\left(1+\left(l^{\prime}-1\right) \sigma\right) /\left(l^{\prime} \kappa-l^{\prime}+1\right)<1 / \kappa$ by $l^{\prime} \geqq 2$ and $\kappa \geqq 2$.

Proof. Set

$$
\tilde{J}_{v+1} \equiv \tilde{J}_{v+1}\left(t, \tilde{t}^{v}, s ; x, \xi\right)=\Phi_{v+1}\left(t, \tilde{t}^{v}, s ; x, \xi\right)-x \cdot \xi .
$$

Then, from (1.25) in [13], (3.13) and (3.5) it follows that

$$
\begin{aligned}
\nabla_{\xi} \tilde{J}_{v+1} & =\nabla_{\xi} \phi_{v+1}\left(t_{v}, s ; X_{v}^{v}, \xi\right)-x \\
& =\left(\nabla_{\xi} \phi_{v+1}\left(t_{v}, s ; X_{v}^{v}, \xi\right)-X_{v}^{v}\right)+\left(X_{v}^{v}-x\right) \\
& =\sum_{m=1}^{v+1} g\left(X_{v}^{m-1}\right)^{l^{\prime}} \int_{t_{m}}^{t_{m-1}} \theta^{l} \nabla_{\xi}\left(\lambda_{m}^{0}\left(\theta, x, \nabla_{x} \phi_{m}\left(\theta, t_{m} ; X_{v}^{m-1}, \Xi_{v}^{m}\right)\right)\right) d \theta
\end{aligned}
$$

and similarly it follows that

$$
\nabla_{x} \tilde{J}_{v+1}=\sum_{m=1}^{v+1} \nabla_{x}\left(g\left(X_{v}^{m-1}\right)^{l^{\prime}} \int_{t_{m}}^{t_{m-1}} \theta^{l} \lambda_{m}^{0}\left(\theta, x, \nabla_{x} \phi_{m}\left(\theta, t_{m} ; X_{v}^{m-1}, \Xi_{v}^{m}\right)\right) d \theta\right)
$$


Hence, using (2.12) in [22] and Lemma 3.6 we have for $\alpha+\beta \neq 0$

$$
\begin{aligned}
\left|\partial_{\xi}^{\alpha} \partial_{x}^{\beta} \tilde{J}_{v+1}\right| \leqq & \sum_{m=1}^{v+1} \sum \frac{\alpha ! \beta !}{\alpha^{\prime} ! \alpha_{1} ! \ldots \alpha_{j} ! \beta^{\prime} ! \beta_{1} ! \ldots \beta_{j} !} g\left(X_{v}^{m-1}\right)^{l^{\prime}-j} \\
& \times\left|\left\{\prod_{j^{\prime}=1}^{j} \partial_{\xi}^{\alpha_{j^{\prime}}} \partial_{x}^{\beta_{j^{\prime}}} g\left(X_{v}^{m-1}\right)\right\} \int_{t_{m}}^{t_{m-1}} \theta^{l} \partial_{\xi}^{\alpha^{\prime}} \partial_{x}^{\beta^{\prime}} \lambda_{m}^{0} d \theta\right| \\
\leqq & M^{-|\alpha+\beta|} \max _{1 \leqq j \leqq|\alpha+\beta|}\{(|\alpha+\beta|-1) ! \\
& \left.\times(|\alpha+\beta|-j) !^{\kappa^{-1}}\left(t^{l+1} \mu\right)^{1-j \mid l^{\prime}}\langle\xi\rangle^{\sigma+j \delta-|\alpha|}\right\}
\end{aligned}
$$

where the second summation in the second member of (3.15) is taken over all $\left(j ; \alpha^{\prime}, \alpha_{1}, \ldots, \alpha_{j}, \beta^{\prime}, \beta_{1}, \ldots, \beta_{j}\right)$ such that $0 \leqq j \leqq l^{\prime}, \alpha^{\prime}+\alpha_{1}+\cdots+\alpha_{j}=\alpha, \beta^{\prime}+$ $\beta_{1}+\cdots+\beta_{j}=\beta$, and $\alpha_{j^{\prime}}+\beta_{j^{\prime}} \neq 0\left(j^{\prime}=1, \ldots, j\right)$. Now, we set

$$
\tilde{J}_{v+1, \alpha, \beta}=\exp \left(-i \tilde{J}_{v+1}\right) \partial_{\xi}^{\alpha} \partial_{x}^{\beta} \exp \left(i \tilde{J}_{v+1}\right)
$$

and use the induction on $|\alpha+\beta|$. Then, since we have for $(\alpha, \beta) \neq 0$

$$
\tilde{J}_{v+1, \alpha, \beta}=\partial_{\xi}^{\alpha^{\prime \prime}} \partial_{x}^{\beta^{\prime \prime}} \tilde{J}_{v+1, \alpha-\alpha^{\prime \prime}, \beta-\beta^{\prime \prime}}+i \tilde{J}_{v+1, \alpha-\alpha^{\prime \prime}, \beta-\beta^{\prime \prime}} \partial_{\xi}^{\alpha^{\prime \prime}} \partial_{x}^{\beta^{\prime \prime}} \tilde{J}_{v+1}
$$

with some $\left(\alpha^{\prime \prime}, \beta^{\prime \prime}\right)$ satisfying $\alpha^{\prime \prime} \leqq \alpha, \beta^{\prime \prime} \leqq \beta$ and $\left|\alpha^{\prime \prime}+\beta^{\prime \prime}\right|=1$, we can prove from (3.15)

$$
\begin{aligned}
\left|\partial_{\xi}^{\alpha^{\prime}} \partial_{x}^{\beta^{\prime}} \tilde{J}_{v+1, \alpha, \beta}\right| \leqq & M_{1}^{-|\alpha+\beta|} M_{2}^{-\left|\alpha^{\prime}+\beta^{\prime}\right|} \\
& \times \max _{1 \leqq m \leqq|\alpha+\beta|} \max \left\{\left(\left|\alpha+\beta+\alpha^{\prime}+\beta^{\prime}\right|-m\right) !\right. \\
& \left.\times\left(\left|\alpha+\beta+\alpha^{\prime}+\beta^{\prime}\right|-j\right) !^{\kappa-1}\left(t^{l+1} \mu\right)^{m-j / l^{\prime}}\langle\xi\rangle^{m \sigma+j \delta-\left|\alpha+\alpha^{\prime}\right|}\right\}
\end{aligned}
$$

for $\left(t, \tilde{t}^{v}, s\right)$ satisfying $(3.10)$, where $\mu=\mu(x, \xi ; \zeta)$ and the second maximum in (3.16) is taken over all $j$ satisfying $m \leqq j \leqq \min \left(\left|\alpha+\beta+\alpha^{\prime}+\beta^{\prime}\right|, m l^{\prime}\right)$. Hence, we have

$$
\begin{aligned}
\left|\partial_{\xi}^{\alpha} \partial_{x}^{\beta} \exp \left(i \tilde{J}_{v+1}\right)\right| \leqq & M_{1}^{-|\alpha+\beta|} \max _{1 \leqq m \leqq|\alpha+\beta|} \max _{j}\{(|\alpha+\beta|-m) ! \\
& \left.\times(|\alpha+\beta|-j) !^{\kappa-1}\left(t^{l+1} \mu\right)^{m-j / l^{\prime}}\langle\xi\rangle^{m \sigma+j \delta-|\alpha|}\right\}
\end{aligned}
$$

for $\left(t, \tilde{t}^{v}, s\right)$ satisfying (3.10). Here and in the next, $\underset{j}{\max }$ means that we take maximum over all $j$ satisfying $m \leqq j \leqq \min \left(|\alpha+\beta|, m l^{\prime}\right)$. From (5) it follows that $(\sigma+\delta) /\left(\kappa-1+1 / l^{\prime}\right) \leqq \sigma^{\prime}$. Hence, using (3.17) we can prove

$$
\begin{aligned}
\left|\partial_{\xi}^{\alpha} \partial_{x}^{\beta} \exp \left[i\left(\Phi_{v+1}-x \cdot \xi\right)\right]\right| \leqq & M_{1}^{-|\alpha+\beta|} \max _{1 \leqq m \leqq|\alpha+\beta|} \max _{j}\{(|\alpha+\beta|-m) ! \\
& \left.\times(|\alpha+\beta|-j) !^{\kappa-1}\left(t^{l+1} \mu\right)^{m-j / l^{\prime}}\langle\xi\rangle^{m \sigma+j \delta-|\alpha|}\right\}
\end{aligned}
$$




$$
\begin{aligned}
\leqq & M_{3, \varepsilon}^{-|\alpha+\beta|} \max _{1 \leqq m \leqq|\alpha+\beta|} \max _{j}\{(|\alpha+\beta|-m) ! \\
& \times(|\alpha+\beta|-j) !^{\kappa-1}\left[m-j / l^{\prime}\right] !\left[(m \sigma+j \delta) / \sigma^{\prime}\right] ! \\
& \left.\times\langle\xi\rangle^{-|\alpha|} \exp \left[\varepsilon t^{l+1} \mu(x, \xi)+\langle\xi\rangle^{\sigma^{\prime}}\right]\right\} \\
\leqq & M_{4, \varepsilon}^{-|\alpha+\beta|} \alpha !^{\kappa} \beta !^{\kappa}\langle\xi\rangle^{-|\alpha|} \exp \left[\varepsilon t^{l+1} \mu(x, \xi)+\langle\xi\rangle^{\sigma^{\prime}}\right] .
\end{aligned}
$$

Hence, we have (3.14).

Q.E.D.

\section{§4. Systemization and Perfectly Diagonalization}

In this section we reduce the Cauchy problem (8) of (6) to a system equivalent to (8). In order to simplify the notation below, we write $p(t, x, \xi ; \zeta)$ simply by $p(t, x, \xi)$. We also omit to describe the terms of regularizers and the equality means that it holds modulo regularizers unless otherwise stated.

First, we factorize the operator $L$ of $(6)$. Let $\lambda_{+}(t, x, \xi)$ be characteristic roots of $L$, which is defined by (3.4). Then, from Proposition 1.4 there exists a symbol $b_{1}(t, x, \xi)$ in $S W F_{1, \delta, G(\kappa)}[0,0,0]$ such that

$$
\begin{aligned}
L= & \left(D_{t}-\lambda_{-}\left(t, X, D_{x}\right)\right)\left(D_{t}-\lambda_{+}\left(t, X, D_{x}\right)\right) \\
& +t^{k} g(x)^{k^{\prime}} b_{0}\left(t, X, D_{x}\right)+b_{1}\left(t, X, D_{x}\right),
\end{aligned}
$$

where

$$
\begin{aligned}
b_{0}(t, x, \xi)= & \sum_{j=1}^{n} a_{j}(x, \xi) \xi_{j}+t^{l-k} g(x)^{l^{\prime}-k^{\prime}} \sum_{|\alpha|=1} \lambda_{0}^{(\alpha)}(t, x, \xi) \lambda_{+(\alpha)}(t, x, \xi) \\
& -i l t^{l-k-1} g(x)^{l^{\prime}-k^{\prime}} \lambda_{0}(t, x, \xi)+t^{l-k} g(x)^{l^{\prime}-k^{\prime}} D_{t} \lambda_{0}(t, x, \xi),
\end{aligned}
$$

which belongs to $\stackrel{\circ}{S}_{1, \delta, G(\kappa)}[1,0,0]$. Now, we set

$$
b(t, x, \xi)=t^{k} g(x)^{k^{\prime}} b_{0}(t, x, \xi) /(2 \tilde{\lambda}(t, x, \xi))
$$

with $\tilde{\lambda}(t, x, \xi)$ in (3.6). Then, from (3.7) we have

$$
\left\{\begin{array}{l}
\text { i) } b(t, x, \xi) \in \stackrel{\circ}{S}_{1, \delta, G(\kappa)}[\sigma, 0,-1], \\
\text { ii) } b_{(\beta)}(t, x, \xi) \in \stackrel{\circ}{S_{1, \delta, G(\kappa)}}\left[\sigma+\delta,-1 / l^{\prime},-1\right] \quad(|\beta|=1),
\end{array}\right.
$$

because from (4) and $\omega=1 /(l+1)$ we have

$$
\left\{\begin{array}{l}
(1-\sigma)\left(1-k^{\prime} / l^{\prime}\right) \leqq \sigma, \\
\omega(l-k-1)-\sigma /(1-\sigma) \leqq 0, \\
\langle\xi\rangle^{-1} \leqq C\left(\mu_{\mid \zeta=1}\right)^{-1 /(1-\sigma)}
\end{array}\right.
$$

and hence we have 


$$
\begin{aligned}
|b(t, x, \xi)| & \leqq C t^{k}\left(g(x)^{l^{\prime}}\langle\xi\rangle^{1-\sigma}\right)^{k^{\prime} / l^{\prime}}\langle\xi\rangle^{1-(1-\sigma) k^{\prime} / l^{\prime}} h^{-l} \mu^{-1}\langle\xi\rangle^{-\sigma} \\
& \leqq C h^{k-l} \mu^{-1}\left(g(x)^{l^{\prime}}\langle\xi\rangle^{1-\sigma}\right)^{k^{\prime} / l^{\prime}}\langle\xi\rangle^{(1-\sigma)\left(1-k^{\prime} / l^{\prime}\right)} \\
& \leqq C h^{-1} \mu^{\omega(l-k-1)-1}\left(\mu_{\mid \zeta=1}\right)^{m}\langle\xi\rangle^{\sigma} \\
& \leqq C h^{-1}\langle\xi\rangle^{\sigma}
\end{aligned}
$$

with a constant $C$ independent of $\zeta$, where $m=k^{\prime} / l^{\prime}-\left\{\sigma-(1-\sigma)\left(1-k^{\prime} / l^{\prime}\right)\right\} /$ $(1-\sigma) \leqq 1-\omega(l-k-1)$. Now, we write (4.1) in the form

$$
\begin{aligned}
L= & \left(D_{t}-\lambda_{-}\left(t, X, \mathbb{D}_{x}\right)-b\left(t, \mathbb{X}, \mathbb{D}_{x}\right)\right) \\
& \times\left(\mathbb{D}_{t}-\lambda_{+}\left(t, \mathbb{X}, \mathbb{D}_{x}\right)+b\left(t, \mathbb{X}, \mathbb{D}_{x}\right)\right) \\
& +b_{2}\left(t, \mathbb{X}, \mathbb{D}_{x}\right)+\tilde{r}\left(t, \mathbb{X}, \mathbb{D}_{x}\right)
\end{aligned}
$$

with

$$
\begin{aligned}
b_{2}(t, x, \xi)= & -D_{t} b(t, x, \xi)-t^{l} g(x)^{l^{\prime}}\left[\lambda_{0} \circ b\right]_{\operatorname{Rem}(1)}(t, x, \xi) \\
& -\left[b \circ \lambda_{+}\right]_{\operatorname{Rem}(1)}(t, x, \xi) \\
& +\sigma_{M}\left(b\left(t, X, D_{x}\right)^{2}\right)+b_{1}(t, x, \xi)
\end{aligned}
$$

and

$$
\tilde{r}(t, x, \xi)=2 b(t, x, \xi)\left\{\tilde{\lambda}(t, x, \xi)-t^{l} g(x)^{l^{\prime}} \lambda_{0}(t, x, \xi)\right\} .
$$

Here, for symbols $p_{j}(t, x, \xi), j=1,2$, we denote $\left[p_{1} \circ p_{2}\right]_{\operatorname{Rem}(1)}(t, x, \xi)=$ $\sigma_{M}\left(\mathbb{P}_{1}(t) P_{2}(t)\right)(x, \zeta)-p_{1}(t, x, \xi) p_{2}(t, x, \xi)$ (see Remark of Proposition 1.2 for the notation $\left.\sigma_{M}(\cdot)\right)$. Now, we use (3.8). Then, we have $\tilde{r} \in \mathscr{H}_{1, \delta, G(\kappa)}[2 \sigma, 2 \omega]$. Moreover, using (4.2)-ii) for the second term in (4.3) and using (4.2)-i) for other terms we find that $b_{2}(t, x, \xi)$ belongs to $S W F_{1, \delta, G(x)}[2 \sigma, 0,-2]$.

Let $h(t, x, \xi) \equiv h(t, x, \xi ; \zeta)$ be a symbol in (3.1) and $h\left(t, X, D_{x}\right)^{-1}$ be the inverse operator constructed in Lemma 3.3. Here and in what follows we assume $\zeta \geqq \zeta_{1}$. For a function $u(t, x)$ we set $U(t, x)={ }^{t}\left(u_{1}(t, x), u_{2}(t, x)\right)$ with $u_{1}(t, x)=h\left(t, X, D_{x}\right)^{-1}\left\langle D_{x}\right\rangle^{\sigma} u \quad$ and $\quad u_{2}(t, x)=\left(D_{t}-\lambda_{+}\left(t, \mathbb{X}, D_{x}\right)+b\left(t, \mathbb{X}, \mathbb{D}_{x}\right)\right) u$. Then, by the same discussion in $[11]$, we can prove that solving the Cauchy problem (8) for (6) is equivalent to solving the Cauchy problem

$$
\left\{\begin{array}{l}
\mathscr{L} U=0, \\
U(s)=U_{0}
\end{array}\right.
$$

for

$$
\begin{aligned}
\mathscr{L}=\mathbb{D}_{t}-\mathscr{D}(t) & +\left(\begin{array}{cc}
b\left(t, X, D_{x}\right)-b_{3}\left(t, X, D_{x}\right) & -h^{-1}\left\langle\mathbb{D}_{x}\right\rangle^{\sigma} \\
b_{4}\left(t, \mathbb{X}, \mathbb{D}_{x}\right) & -b\left(t, \mathbb{X}, \mathbb{D}_{x}\right)
\end{array}\right) \\
& +\left(\begin{array}{cc}
0 & 0 \\
\tilde{R}\left\langle\mathbb{D}_{x}\right\rangle^{-\sigma} h & 0
\end{array}\right)+\mathbb{R}_{\infty, 1}(t),
\end{aligned}
$$


where

$$
\begin{gathered}
\mathscr{D}(t)=\left(\begin{array}{cc}
\lambda_{+}\left(t, X, D_{x}\right) & 0 \\
0 & \lambda_{-}\left(t, X, D_{x}\right)
\end{array}\right), \\
b_{3}(t, x, \xi)=\sigma_{M}\left(\left[D_{t}-\lambda_{+}\left(t, X, D_{x}\right)+b\left(t, X, D_{x}\right), h^{-1}\left\langle D_{x}\right\rangle^{\sigma}\right]\left\langle D_{x}\right\rangle^{-\sigma} h\right) \\
\left(\in S W F_{1, \delta, G(\kappa)}[\sigma, 0,-1]\right), \\
b_{4}(t, x, \xi)=\sigma_{M}\left(b_{2}\left(t, X, D_{x}\right)\left\langle D_{x}\right\rangle^{-\sigma} h\right)
\end{gathered}
$$

with $h=h\left(t, X, D_{x}\right), \widetilde{R}=\tilde{r}\left(t, X, D_{x}\right)$, and $R_{\infty, 1}(t)$ is a matrix of regularizers. Summing up we have proved

Proposition 4.1. Let $\mathscr{L}$ be a hyperbolic system defined by (4.6). Then, we can reduce the problem of solving the Cauchy problem (8) is reduced to the problem of solving (4.5) for a system $\mathscr{L}$ of (4.6).

Next, we diagonalize the operator

$$
\begin{aligned}
\mathscr{L}_{1}=D_{t}-\mathscr{D}(t)+ & \left(\begin{array}{ll}
\tilde{b}\left(t, X, D_{x}\right) & -h^{-1}\left\langle D_{x}\right\rangle^{\sigma} \\
b_{4}\left(t, X, D_{x}\right) & -b\left(t, X, D_{x}\right)
\end{array}\right) \\
& \left(\tilde{b}(t, x, \xi)=b(t, x, \xi)-b_{3}(t, x, \xi)\right)
\end{aligned}
$$

perfectly modulo Hermite operators.

Proposition 4.2 (cf. Theorem 2.2 of [17]). Let $\mathscr{L}_{1}$ be a hyperbolic system of the form (4.8). Then, there exist a diagonal pseudo-differential operator $F(t)$ with the symbol in $S W F_{1, \delta, G(\kappa)}[\sigma, 0,-1]$ and a pseudo-differential operator $P(t)$ with a symbol in $S W F_{1, \delta, G(\kappa)}[0,-1,-(l+1)]$ such that

$$
\mathscr{L}_{1}(I+P(t))=(I+P(t))\left(D_{t}-\mathscr{D}(t)+F(t)\right)+\tilde{\widetilde{R}}(t)+R_{\infty, 2}(t),
$$

where $\tilde{\widetilde{R}}(t)$ and $R_{\infty, 2}(t)$ are matrices of pseudo-differential operators with the symbols in $\mathscr{H}_{1, \delta, G(\kappa)}[\sigma, \omega]$ and $\mathscr{R}_{G(\kappa)}$, respectively.

Proof. Set

$$
\begin{aligned}
& B \equiv B(t)=\left(\begin{array}{cc}
\tilde{b}\left(t, X, D_{x}\right) & 0 \\
0 & -b\left(t, X, D_{x}\right)
\end{array}\right), \\
& B^{\prime} \equiv B^{\prime}(t)=\left(\begin{array}{cc}
0 & -h^{-1}\left\langle D_{x}\right\rangle^{\sigma} \\
b_{4}\left(t, X, D_{x}\right) & 0
\end{array}\right)
\end{aligned}
$$

and we will find an operator $P \equiv P(t)$ with the symbol in $S W F_{1, \delta, G(\kappa)}[0,-1$, $-(l+1)]$ and with zero diagonal elements such that it satisfies

$$
\begin{gathered}
\mathscr{D} P-P \mathscr{D} \equiv P_{t}+B^{\prime}+B P-P B-P B^{\prime} P \\
\bmod \mathscr{H}_{1, \delta, G(\kappa)}[\sigma, \omega]+\mathscr{R}_{G(\kappa)},
\end{gathered}
$$

where $\sigma\left(P_{t}\right)=D_{t} \sigma(P)$. Then, defining a pseudo-differential operator $F(t)$ by 


$$
\sigma(F(t))=B(t)+\sigma_{M}\left(B^{\prime}(t) P(t)\right)
$$

we find that $P(t)$ and $F(t)$ satisfy (4.9) with an Hermite operator $\widetilde{\widetilde{R}}(t)$ and an regularizer $R_{\infty, 2}(t)$.

In order to find $P(t)$ we set

$$
\tilde{\mathscr{D}}=\left(\begin{array}{cc}
\tilde{\lambda}\left(t, X, D_{x}\right) & 0 \\
0 & -\tilde{\lambda}\left(t, X, D_{x}\right)
\end{array}\right)
$$

with $\tilde{\lambda}(t, x, \xi)$ in Lemma 3.5. Assume that $\sigma(\mathbb{P}(t)) \in S W F_{1, \delta, G(\kappa)}[0,-1$, $-(l+1)]$. Then, by $(3.8)$ the relation $(4.10)$ is equivalent to

$$
\begin{gathered}
\tilde{\mathscr{D}} P-P \tilde{\mathscr{D}} \equiv P_{t}+B^{\prime}+B P-P B-P B^{\prime} P \\
\bmod \mathscr{H}_{1, \delta, G(\kappa)}[\sigma, \omega]+\mathscr{R}_{G(\kappa)} .
\end{gathered}
$$

Since $\sigma(B(t))$ and $\sigma_{M}\left(B^{\prime}(t)\right)$ belong to $S W F_{1, \delta, G(\kappa)}[\sigma, 0,-1]$, they have formal symbols $\sum \sigma\left(B_{j}(t)\right)$ and $\sum \sigma\left(B_{j}^{\prime}(t)\right)$. Now, we find $\sigma(P(t))$ as a formal $\operatorname{sum} \sum_{v, m} \sigma\left(P_{v, m}\right)$ with $\sigma\left(P_{v, m}\right) \in S_{1, \delta, G(\kappa)}[-v(1-\delta),-(m+1),-(m+1)(l+1)]$ satisfying

$$
\begin{aligned}
\sigma\left(P_{0,0}\right)= & \sigma(\tilde{\Lambda})^{-1} \sigma\left(B_{0}^{\prime}\right) \\
\sigma\left(P_{0, m}\right)= & \sigma(\tilde{\Lambda})^{-1}\left\{D_{t} \sigma\left(P_{0, m-1}\right)+\sigma\left(B_{0}\right) \sigma\left(P_{0, m-1}\right)-\sigma\left(P_{0, m-1}\right) \sigma\left(B_{0}\right)\right. \\
& \left.-\sum_{m^{\prime}+m^{\prime \prime}=m-2} \sigma\left(P_{0, m^{\prime}}\right) \sigma\left(B_{0}^{\prime}\right) \sigma\left(P_{0, m^{\prime \prime}}\right)\right\} \quad(m \geqq 1)
\end{aligned}
$$

$$
\begin{gathered}
\sigma\left(P_{v, 0}\right)=\sigma(\tilde{\Lambda})^{-1}\left\{\sigma\left(B_{v}^{\prime}\right)+\sum_{\substack{v^{\prime}+|\gamma|=v \\
\gamma \neq 0}} \frac{1}{\gamma !}\left\{\sigma\left(P_{v^{\prime}, 0}\right)^{(\gamma)} \sigma(\tilde{\mathscr{D}})_{(\gamma)}-\sigma(\tilde{\mathscr{D}})^{(\gamma)} \sigma\left(P_{v^{\prime}, 0}\right)_{(\gamma)}\right\}\right. \\
(v \geqq 1)
\end{gathered}
$$

and

$$
\begin{aligned}
\sigma\left(P_{v, m}\right)=\sigma(\tilde{\Lambda})^{-1}\left[D_{t} \sigma\left(P_{v, m-1}\right)\right. & \\
+ & \sum_{v^{\prime}+v^{\prime \prime}+|\gamma|=v} \frac{1}{\gamma !}\left\{\sigma\left(B_{v^{\prime}}\right)^{(\gamma)} \sigma\left(P_{v^{\prime \prime}, m-1}\right)_{(\gamma)}-\sigma\left(P_{v^{\prime}, m-1}\right)^{(\gamma)} \sigma\left(B_{v^{\prime \prime}}\right)_{(\gamma)}\right\} \\
& -\sum_{\substack{v^{1}+v^{2}+v^{3}+\left|\gamma^{1}\right| m^{\prime}+m^{\prime \prime}=m-2 \\
+\left|\gamma^{2}\right|+\left|\gamma^{3}\right|=v}} \frac{1}{\gamma^{1} ! \gamma^{2} ! \gamma^{3 !}} \sigma\left(P_{v^{1}, m^{\prime}}\right)^{\left(\gamma^{1}+\gamma^{2}\right)} \\
& \times \sigma\left(B_{v^{2}}^{\prime}\right)_{\left(\gamma^{1}\right)}^{\left(\gamma^{3}\right)} \sigma\left(P_{v^{3}, m^{\prime \prime}}\right)^{\left(\gamma^{2}+\gamma^{3}\right)}
\end{aligned}
$$




$$
\begin{array}{r}
\left.+\sum_{\substack{v^{\prime}+|\gamma|=v \\
\gamma \neq 0}} \frac{1}{\gamma !}\left\{\sigma\left(P_{v^{\prime}, m}\right)^{(\gamma)} \sigma(\tilde{\mathscr{D}})_{(\gamma)}-\sigma(\tilde{\mathscr{D}})^{(\gamma)} \sigma\left(P_{v^{\prime}, m}\right)_{(\gamma)}\right\}\right] \\
(v \geqq 1, m \geqq 1) .
\end{array}
$$

Here, when $m=1$, we mean that the last term in (4.12) and the third term in (4.14) do not appear, and

$$
\tilde{\Lambda}=\left(\begin{array}{cc}
2 \tilde{\lambda}\left(t, X, D_{x}\right) & 0 \\
0 & -2 \tilde{\lambda}\left(t, X, D_{x}\right)
\end{array}\right) .
$$

Then, as in Section 6 of [22] we find that $\sigma\left(P_{v, m}\right)$ satisfy

$$
\begin{aligned}
\left|\partial_{t}^{\gamma} \sigma\left(P_{v, m}\right)_{(\beta)}^{(\alpha)}\right| \leqq & C M_{1}^{|\alpha+\beta|+\gamma+v+m} \alpha ! \gamma ! m ! \\
& \times\left((|\beta|+v) !^{\kappa}+(|\beta|+v) !^{\lfloor(1-\delta)}\langle\xi\rangle^{\delta(|\beta|+v)}\right) \\
& \times h(t, x, \xi)^{-\gamma}\langle\xi\rangle^{-|\alpha|-v}\left(h^{l+1} \mu\right)^{-m-1}
\end{aligned}
$$

by using a formal norm

$$
\begin{aligned}
\|\left\{\sigma\left(P_{v, m}\right\}, M \| \mid=\right. & \sum_{\alpha, \beta, \gamma, v, m} \frac{2(2 n)^{-v} v !}{(v+|\alpha|) !(m+v+|\beta|+\gamma) !} \\
& \times M^{2 m+2 v+|\alpha|+|\beta|+\gamma} \\
& \times \sup \left\{\left|\partial_{t}^{\gamma} \sigma\left(P_{v, m}\right)_{(\beta)}^{(\alpha)}\right|\right. \\
& \times\left((|\beta|+v)^{\kappa-1}+(|\beta|+v)^{\kappa(1-\delta)-1}\langle\xi\rangle^{\delta}\right)^{-(|\beta|+v)} \\
& \left.\times\langle\xi\rangle^{v+|\alpha|}\left(h^{l+1} \mu\right)^{m+1} h^{\gamma}\right\} \quad(\mathrm{cf} .[4],[7]) .
\end{aligned}
$$

First, we use discussion in pp. $314-317$ of [4]. Then, for a sequence $\left\{s_{m}\right\}$ of $(2 \times 2)$-matrices $s_{m}$ of complex numbers satisfying

$$
\left\|\left\{s_{m}\right\}\right\| \equiv\left\{\sum_{m=0}^{\infty}\left|s_{m}\right|^{2} M_{2}{ }^{2 m} m !^{-4}\right\}^{1 / 2}<\infty
$$

we find a matrix $\psi(\theta)$ satisfying

$$
\left\{\begin{array}{l}
\left|\partial_{\theta}^{j} \psi(\theta)\right| \leqq C\left\|\left\{s_{m}\right\}\right\| M_{3}^{-j} j !|\theta|^{-j} \quad(\theta \neq 0), \\
\left|\partial_{\theta}^{j}\left(\psi(\theta)-\sum_{m=0}^{N-1} \frac{\theta^{m}}{m !} s_{m}\right)\right| \leqq C\left\|\left\{s_{m}\right\}\right\| M_{3}^{-(j+N)} j ! N !|\theta|^{N-j} \quad(\theta \neq 0) .
\end{array}\right.
$$

For a fixed $v$ we apply this result to $s_{m}=\sigma\left(P_{v, m}\right)(t, x, \xi ; \zeta)\left(h(t, x, \xi ; \zeta)^{l+1} \times\right.$ $\mu(x, \xi ; \zeta))^{m} m$ ! with a parameter $t, x, \xi$ and $\zeta$. Then, we find a function $\psi_{v}(\theta ; t, x, \xi) \equiv \psi_{v}(\theta ; t, x, \xi ; \zeta)$ satisfying 
$\left|\partial_{\theta}^{j} \partial_{t}^{\gamma} \partial_{\xi}^{\alpha} \partial_{x}^{\beta} \psi_{v}\right| \leqq C M^{-(|\alpha+\beta|+\gamma+v+j)} \alpha ! \gamma ! j !$

$$
\begin{aligned}
& \times\left((|\beta|+v) !^{\kappa}+(|\beta|+v) !^{\kappa(1-\delta)}\langle\xi\rangle^{\delta(|\beta|+v}\right)\left(h^{l+1} \mu\right)^{-1} \\
& \times\langle\xi\rangle^{-|\alpha|-v} h^{-\gamma}|\theta|^{-j} \quad \text { for } \quad \theta \neq 0, \\
& \left|\partial_{\theta}^{j} \partial_{t}^{\gamma} \partial_{\xi}^{\alpha} \partial_{x}^{\beta}\left\{\psi_{v}(\theta ; t, x, \xi)-\sum_{m=0}^{N-1} \frac{\theta^{m}}{m !} s_{m}(t, x, \xi)\right\}\right| \\
& \leqq C M^{-(|\alpha+\beta|+\gamma+j+\nu+N)} \alpha ! \gamma ! j ! N ! \\
& \times\left((|\beta|+v) !^{\kappa}+(|\beta|+v) !^{\kappa(1-\delta)}\langle\xi\rangle^{\delta(|\beta|+v)}\right)\left(h^{l+1} \mu\right)^{-1} \\
& \times\langle\xi\rangle^{-|\alpha|-v} h^{-\gamma}|\theta|^{N-j} \text { for } \theta \neq 0 .
\end{aligned}
$$

Define pseudo-differential operators $\mathbb{P}_{v}$ as

$$
\sigma\left(P_{v}\right)=\psi_{v}\left(1 /\left\{h(t, x, \xi)^{l+1} \mu(x, \xi)\right\} ; t, x, \xi\right) .
$$

Then, $\sigma\left(P_{v}\right)$ satisfy

$$
\left\{\begin{aligned}
\left|\partial_{t}^{\gamma} \sigma\left(P_{v}\right)_{(\beta)}^{(\alpha)}\right| \leqq & C M^{-(|\alpha+\beta|+\gamma+v)} \alpha ! \gamma ! \\
& \times\left((|\beta|+v) !^{\kappa}+(|\beta|+v) !^{\kappa(1-\delta)}\langle\xi\rangle^{\delta(|\beta|+v)}\right)\left(h^{l+1} \mu\right)^{-1} \\
& \times\langle\xi\rangle^{-|\alpha|-v} h^{-\gamma} \\
\mid \partial_{t}^{\gamma} \partial_{\xi}^{\alpha} \partial_{x}^{\beta}\left\{\sigma\left(P_{v}\right)\right. & \left.-\sum_{m=0}^{N-1} \sigma\left(P_{v, m}\right)\right\} \mid \\
\leqq & C M^{-(\mid \alpha+\beta+\gamma+v+N)} \alpha ! \gamma ! N ! \\
& \times\left((|\beta|+v) !^{\kappa}+(|\beta|+v) !^{\kappa(1-\delta)}\langle\xi\rangle^{\delta(|\beta|+v)}\right) \\
& \times\langle\xi\rangle^{-|\alpha|-v} h^{-\gamma}\left(h^{l+1} \mu\right)^{-1-N}
\end{aligned}\right.
$$

Now, we set

$$
\begin{aligned}
\sigma\left(R_{0}\right)= & \left\{\sigma(\tilde{D}) \sigma\left(\mathbb{P}_{0}\right)-\sigma\left(\mathbb{P}_{0}\right) \sigma(\tilde{\mathscr{D}})\right\}-\left\{D_{t} \sigma\left(\mathbb{P}_{0}\right)+\sigma\left(\mathbb{B}_{0}^{\prime}\right)\right. \\
& \left.+\sigma\left(B_{0}\right) \sigma\left(\mathbb{P}_{0}\right)-\sigma\left(\mathbb{P}_{0}\right) \sigma\left(B_{0}\right)-\sigma\left(\mathbb{P}_{0}\right) \sigma\left(B_{0}^{\prime}\right) \sigma\left(\mathbb{P}_{0}\right)\right\} \\
\sigma\left(\mathbb{R}_{v}\right)= & \left\{\sigma(\tilde{\mathscr{D}}) \sigma\left(\mathbb{P}_{v}\right)-\sigma\left(\mathbb{P}_{v}\right) \sigma(\tilde{\mathscr{D}})\right\}-\left\{D_{t} \sigma\left(\mathbb{P}_{v}\right)+\sigma\left(\mathbb{B}_{v}^{\prime}\right)\right. \\
& +\sum_{v^{\prime}+v^{\prime \prime}+|\gamma|=v} \frac{1}{\gamma !}\left\{\sigma\left(B_{v^{\prime}}\right)^{(\gamma)} \sigma\left(\mathbb{P}_{v^{\prime \prime}}\right)_{(\gamma)}-\sigma\left(P_{v^{\prime}}\right)^{(\gamma)} \sigma\left(\mathbb{B}_{v^{\prime \prime}}\right)_{(\gamma)}\right\} \\
& -\sum_{v^{1}+v^{2}+v^{3}+\left|\gamma^{1}\right|+\left|\gamma^{2}\right|+\left|\gamma^{3}\right|=v} \frac{1}{\gamma^{1} ! \gamma^{2} ! \gamma^{3} !} \sigma\left(\mathbb{P}_{v^{1}}\right)^{\left(\gamma^{1}+\gamma^{2}\right)} \sigma\left(\mathbb{B}_{v^{2}}^{\prime}\right)_{\left(\gamma^{1}\right)}^{\left(\gamma^{3}\right)} \sigma\left(\mathbb{P}_{v^{3}}\right)_{\left(\gamma^{2}+\gamma^{3}\right)} \\
& \left.+\sum_{\substack{v^{\prime}+|\gamma|=v \\
\gamma \neq 0}} \frac{1}{\gamma^{\prime} !}\left\{\sigma\left(\mathbb{P}_{v^{\prime}}\right)^{(\gamma)} \sigma(\tilde{\mathscr{D}})_{(\gamma)}-\sigma(\tilde{\mathscr{D}})^{(\gamma)} \sigma\left(\mathbb{P}_{v^{\prime}}\right)^{(\gamma)}\right\}\right\} \quad(v \geqq 1) .
\end{aligned}
$$


Then, from (4.15) we have

$$
\begin{aligned}
\left|\sigma\left(R_{v}\right)_{(\beta)}^{(\alpha)}\right| \leqq & C M^{-(|\alpha+\beta|+v)} \alpha ! \\
& \times\left((|\beta|+v) !^{\kappa}+(|\beta|+v !)^{\kappa(1-\delta)}\langle\xi\rangle^{\delta(|\beta|+v)}\right) \\
& \times\langle\xi\rangle^{\sigma-|\alpha|-v} \mu^{\omega} \exp \left(-\varepsilon t^{l+1} \mu\right)
\end{aligned}
$$

for an $\varepsilon>0$ independent of $v$. Next, we apply Lemma 1.3 to formal symbols $\Sigma \sigma\left(P_{v}\right)$ and $\Sigma \sigma\left(R_{v}\right)$. Then, we find symbols $\sigma(P)$ in $S W F_{1, \delta, G(\kappa)}[0,-1,-(l+1)]$ and $\sigma(R)$ in $\mathscr{H}_{1, \delta, G(\kappa)}[\sigma, \omega]$ satisfying

$$
\begin{aligned}
&\left|\partial_{t}^{\gamma} \partial_{\xi}^{\alpha} \partial_{x}^{\beta}\left(\sigma(P)-\sum_{v<N} \sigma\left(P_{v}\right)\right)\right| \\
& \leqq C M^{-(|\alpha+\beta|+j+N)} \alpha ! \gamma !\left((|\beta|+N) !^{\kappa}+(|\beta|+N) !^{\kappa(1-\delta)}\langle\xi\rangle^{\delta(|\beta|+N)}\right) \\
& \times\left(h^{l+1} \mu\right)^{-1}\langle\xi\rangle^{-|\alpha|-N} \quad \text { for }\langle\xi\rangle \geqq c(|\alpha|+N)^{\kappa}
\end{aligned}
$$

and

$$
\begin{aligned}
&\left|\partial_{\xi}^{\alpha} \partial_{x}^{\beta}\left(\sigma(R)-\sum_{\nu<N} \sigma\left(R_{v}\right)\right)\right| \leqq C M^{-(|\alpha+\beta|+N)} \alpha !\left((|\beta|+N) !^{\kappa}\right. \\
&\left.+(|\beta|+N) !^{\kappa(1-\delta)}\langle\xi\rangle^{\delta(|\beta|+N)}\right) \\
& \times\langle\xi\rangle^{\sigma-|\alpha|-N} \mu^{\omega} \exp \left(-\varepsilon t^{l+1} \mu\right) \\
& \text { for }\langle\xi\rangle \geqq c(|\alpha|+N)^{\kappa}
\end{aligned}
$$

Consequently, from (4.16)-(4.18) we obtain (4.10)' and (4.9) for a Hermite operator $\widetilde{R}(t)$ and a regularizer $R_{\infty, 2}(t)$.

Q.E.D.

Since $h(t, x, \xi ; \zeta)^{l+1} \mu(t, x ; \zeta) \geqq \zeta$, the formal norm $\|\sigma(P) ; M\|$ of $\sigma(P)(t, x, \xi ; z)$ satisfies

$$
\|\sigma(P) ; M\| \leqq C \zeta^{-1}
$$

if we consider $\sigma(P)$ as a symbol in $S_{1, \delta, G(\kappa)}^{0}$. Hence, using Proposition 1.9 we find an inverse operator $(I+P)^{-1}$ of $I+P$ if $\zeta$ is sufficiently large. We fix such a $\zeta$ till the end of this paper. Then, from (4.8)-(4.9) we have for the system $\mathscr{L}$ of (4.6)

$$
\mathscr{L}(I+P)=(I+P) \mathscr{L}_{2}
$$

with

$$
\begin{aligned}
\mathscr{L}_{2}= & D_{t}-\mathscr{D}(t)+F(t)+(I+P)^{-1}\left\{\tilde{\widetilde{R}}(t)+\left(\begin{array}{cc}
0 & 0 \\
\tilde{R}\left\langle D_{x}\right\rangle^{-\sigma} h & 0
\end{array}\right)(I+P)\right\} \\
& +(I+P)^{-1}\left\{R_{\infty, 2}(t)+R_{\infty, 1}(t)(I+P)\right\}
\end{aligned}
$$


where $\tilde{R}(t)$ and $R_{\infty, 1}(t)$ are operators in (4.6). We note that we used the similar discussion in the proof of Proposition 1.4 in order to obtain the fact that the main symbol of $(I-P)^{-1}$ times an Hermite operator also belongs to $\mathscr{H}_{1, \delta, G(\kappa)}[\sigma, \omega]$.

Considering Proposition 4.1 and (4.19), Theorem 1 is reduced to the following theorem.

Theorem 3. Let $\mathscr{D}(t)$ be (4.7) with $\lambda_{ \pm}(t, x, \xi)$ in (3.4), $F(t)$ be a diagonal matrix of pseudo-differential operators with symbols in $S_{1, \delta, G(\kappa)}[\sigma, 0,-1]$ and $R(t)$ and $R_{\infty}(t)$ be matrices of pseudo-differential operators whose symbols belong to $\mathscr{H}_{1, \delta, G(\kappa)}[\sigma, \omega]$ and $\mathscr{R}_{G(\kappa)}$, respectively. Then, for the Cauchy problem (4.5) of a system

$$
\mathscr{L}=D_{t}-\mathscr{D}(t)+F(t)+R(t)+R_{\infty}(t)
$$

we can construct the fundamental solution $\mathbb{E}(t, s)$ in the form

$$
E(t, s)=\sum_{ \pm} I_{\phi_{ \pm}}(t, s) E_{ \pm}(t, s)+E_{0}(t, s)+\mathbb{E}_{\infty}(t, s)
$$

for $0 \leqq s \leqq t \leqq T_{0}$ with a small constant $T_{0}$ and the symbols $e_{j}(t, s ; x, \xi), j=0$, ,$\pm \infty$, of $E_{j}(t, s)$ satisfy $(10)-(12)$.

\section{\$5. Construction of the Fundamental Solution for a Hyperbolic Operator}

We consider a hyperbolic operator

$$
L=D_{t}-\lambda\left(t, X, D_{x}\right)+f\left(t, X, D_{x}\right),
$$

where $\lambda(t, x, \xi)$ is a real-valued symbol in $S_{1,0, G(\kappa)}^{1}$ and $f(t, x, \xi)$ is a symbol in $\tilde{S}_{1, \delta, G(\kappa)}[\sigma, 0,-1]$ with $\sigma \kappa<1$. Let $\phi(t, s ; x, \xi)$ be a phase function corresponding to $\lambda(t, x, \xi)$ and denote by $\mathbb{I}_{\phi}(t, s)$ the Fourier integral operator with the phase function $\phi(t, s ; x, \xi)$ and the symbol 1 . Set $\rho=1-\delta$. Then, we have

Proposition 5.1. The Cauchy problem for $L$ of (5.1) has a fundamental solution $E(t, s)$ in the form

$$
E(t, s)=I_{\phi}(t, s)\left(\tilde{E}(t, s)+\tilde{E}_{\infty}(t, s)\right) .
$$

In (5.2) $\tilde{E}(t, s)$ is a pseudo-differential operator with the symbol $\tilde{e}(t, s ; x, \xi)$ in $S_{\rho, G(\kappa)}\left[w_{0}\right]$ for

$$
w_{0}(\theta)=\exp \left[C \theta^{\sigma} \log \left\{\left(t \theta^{\omega(1-\sigma)}+1\right) /\left(s \theta^{\omega(1-\sigma)}+1\right)\right\}\right] \quad(C>0)
$$

and $\tilde{E}_{\infty}(t, s)$ is a regularizer in $\mathscr{R}_{G(\kappa)}$.

Proof. We seek $E(t, s)$ in the form

$$
E(t, s)=I_{\phi}(t, s) V(t, s) \text {. }
$$


Operate $L$ to $E(t, s)$. Then, we have

$$
\begin{aligned}
L E(t, s)= & \left(I_{\phi}(t, s)\right)_{t} V(t, s)+I_{\phi}(t, s) V_{t}(t, s) \\
& -\left\{\lambda\left(t, X, D_{x}\right) I_{\phi}(t, s)\right\} V(t, s)+\left\{f\left(t, X, D_{x}\right) I_{\phi}(t, s)\right\} V(t, s),
\end{aligned}
$$

where $\left(I_{\phi}(t, s)\right)_{t}$ is the Fourier integral operator with the symbol $D_{t} \phi(t, s ; x, \xi)$ and $V_{t}(t, s)$ is the pseudo-differential operator with the symbol $D_{t} \sigma(V(t, s))$. Use (2.2) with $N=1, \rho=1$ and $w(\theta)=\theta$ in order to estimate the third term in (5.4). Then, there exist symbols $b_{1}(t, s ; x, \xi)$ in $S_{\rho, \delta, G(\kappa)}^{0}$ and $r_{1}(t, s ; x, \xi)$ in $\mathscr{R}_{G(\kappa)}$ such that

$$
\left(I_{\phi}(t, s)\right)_{t}-\lambda\left(t, X, D_{x}\right) I_{\phi}(t, s)=b_{1, \phi}\left(t, s ; X, D_{x}\right)+r_{1}\left(t, s ; X, D_{x}\right) .
$$

Hence, using Lemma 2.5, Lemma 2.3 and Lemma 2.6 we find symbols $b_{2}(t, s ; x, \xi)$ and $r_{2}(t, s ; x, \xi)$ such that $\left(t+\langle\xi\rangle^{-\omega(1-\sigma)}\right) b_{2} \in S_{\rho, \delta, G(\kappa)}^{\sigma}, r_{2} \in \mathscr{R}_{G(\kappa)}$ and

$$
\begin{aligned}
L E(t, s)= & I_{\phi}(t, s) V_{t}(t, s)+I_{\phi}(t, s) I_{\phi^{*}}(t, s)(P(t, s)+R(t, s))\left\{b_{1, \phi}\left(t, s ; X, D_{x}\right)\right. \\
& \left.+r_{1}\left(t, s ; X, D_{x}\right)+f\left(t, X, D_{x}\right) I_{\phi}(t, s)\right\} V(t, s) \\
= & I_{\phi}(t, s)\left\{V_{t}(t, s)+\left(b_{2}\left(t, s ; X, D_{x}\right)+r_{2}\left(t, s ; X, D_{x}\right)\right) V(t, s)\right\} .
\end{aligned}
$$

Let

$$
B(t, s)=b_{2}\left(t, s ; X, D_{x}\right)+r_{2}\left(t, s ; X, D_{x}\right)
$$

Then, $V(t, s)$ must satisfy

$$
V_{t}(t, s)+B(t, s) V(t, s)=0
$$

Set

$$
\left\{\begin{array}{l}
V_{1}(t, s)=-i \int_{s}^{t} B\left(t^{\prime}, s\right) d t^{\prime}, \\
V_{v+1}(t, s)=-i \int_{s}^{t} B\left(t^{\prime}, s\right) V_{v}\left(t^{\prime}, s\right) d t^{\prime} .
\end{array}\right.
$$

Then, $V(t, s)=I+\sum_{v=1}^{\infty} V_{v}(t, s)$ is a "formal" solution of (5.5).

Now, we estimate symbols of $V_{v+1}(t, s)$. From (5.6) we have

$$
V_{v+1}(t, s)=(-i)^{v+1} \int_{s}^{t} \int_{s}^{t_{1}} \ldots \int_{s}^{t_{v}} B\left(t_{1}, s\right) B\left(t_{2}, s\right) \ldots B\left(t_{v+1}, s\right) d t_{v+1} \ldots d t_{1} .
$$

Hence, modulo regularizers $V_{v+1}(t, s)$ is equal to the pseudo-differential operator $V_{v+1}^{0}(t, s)$ defined by

$$
V_{v+1}^{0}(t, s)=(-i)^{v+1} \int_{s}^{t} \int_{s}^{t_{1}} \ldots \int_{s}^{t_{v}} b_{2}\left(t_{1}, s ; X, D_{x}\right) \ldots b_{2}\left(t_{v+1}, s ; X, D_{x}\right) d t_{v+1} \ldots d t_{1} .
$$


As in the proof of Proposition 1.5 we replace $b_{2}\left(t_{j}, s ; X, \mathbb{D}_{x}\right), \quad j=1$, $\ldots, v$, by $b_{2}^{\prime}\left(t_{j}, s ; \mathbb{X}, \mathbb{D}_{x}, \mathbb{X}^{\prime}\right)$, where $b_{2}^{\prime}\left(t_{j}, s ; x, \xi, x^{\prime}\right)=\left\{\left(1-\Delta_{\xi}\left(\langle\xi\rangle^{2 \delta} \cdot\right) \times(1\right.\right.$ $\left.\left.+\langle\xi\rangle^{2 \delta}\left|x-x^{\prime}\right|^{2}\right)^{-1}\right\}^{[n / 2]+1} b_{2}\left(t_{j}, s ; x, \xi\right)$. Then, since we have

$$
\begin{aligned}
& \int_{s}^{t} \int_{s}^{t_{1}} \ldots \int_{s}^{t_{v}} \prod_{j=1}^{v+1}\left(t_{j}+\theta^{-\omega(1-\sigma)}\right)^{-1} d t_{v+1} \ldots d t_{1} \\
& \quad=\left[\log \left\{\left(t \theta^{\omega(1-\sigma)}+1\right) /\left(s \theta^{\omega(1-\sigma)}+1\right)\right\}\right]^{v+1} /(v+1) !,
\end{aligned}
$$

$V_{v+1}^{0}(t, s)$ is expressed by a multiple symbol

$$
\begin{aligned}
p\left(t, s ; x, \tilde{\xi}^{v}, \tilde{x}^{v}, \xi\right)= & \int_{s}^{t} \int_{s}^{t_{1}} \cdots \int_{s}^{t_{v}} \prod_{j=1}^{v} b_{2}\left(t_{j}, s ; x^{j-1}, \xi^{j}, x^{j}\right) \\
& \times b_{2}\left(t_{v+1}, s ; x^{v}, \xi\right) d t_{v+1} \ldots d t_{1} \quad\left(x^{0}=x, \xi^{v+1}=\xi\right)
\end{aligned}
$$

and it satisfies (1.20) with

$$
w_{v+1}(\theta)=\left[\theta^{\sigma} \log \left\{\left(t \theta^{\omega(1-\sigma)}+1\right) /\left(s \theta^{\omega(1-\sigma)}+1\right\}\right]^{v+1} /(v+1) !\right.
$$

and with $C$ replaced by $C_{1}^{v+1}$ for a constant $C_{1}$. Note that $w_{v+1}(\theta)$ satisfies (1.19) with $W_{v+1, \varepsilon}=\left(C_{\varepsilon}\right)^{v+1}(v+1) !^{-1+\sigma^{\prime} \kappa}$ for a $\sigma^{\prime}$ satisfying $\sigma<\sigma^{\prime}<1 / \kappa$. Hence, applying Lemma $1.6, V_{v+1}^{0}(t, s)$ has the form

$$
V_{v+1}^{0}(t, s)=v_{v+1}\left(t, s ; X, \mathbb{D}_{x}\right)+v_{v+1, \infty}\left(t, s ; \mathbb{X}, \mathbb{D}_{x}\right)
$$

with

$$
\begin{aligned}
&\left|v_{v+1(\beta)}^{(\alpha)}\right| \leqq C^{v+1} M^{-|\alpha+\beta|} \\
& \times\left(|\alpha+\beta| !^{\kappa}+|\alpha+\beta| !^{\kappa \rho}\langle\xi\rangle^{(1-\rho)|\alpha+\beta|}\right)\langle\xi\rangle^{-|\alpha|} W_{v+1}(2\langle\xi\rangle) \\
&\left|v_{v+1, \infty(\beta)}\right| \leqq C_{\alpha} C_{2}^{v+1} M^{-|\beta|} \beta !^{\kappa}(v+1) !^{-1+\sigma^{\prime} \kappa} \exp \left(-\varepsilon\langle\xi\rangle^{1 / \kappa}\right) \\
&\left(\sigma<\sigma^{\prime}<1 / \kappa, \varepsilon>0\right) .
\end{aligned}
$$

Repeating the above discussion again we can prove that $\sigma\left(\mathbb{V}_{v+1}(t, s)-\mathbb{V}_{v+1}^{0}(t, s)\right)$ has also an estimate (5.8). Hence, the sum $\sum_{v=0}^{\infty} \mathbb{V}_{v}(t, s)$ has a meaning and $E(t, s)$ can be written in the form (5.2) with the desired symbol $\tilde{e}(t, s ; x, \xi)=$ $\sigma(\widetilde{E}(t, s))$ in $S_{\rho, G(\kappa)}\left[w_{0}\right]$ for $w_{0}(\theta)$ in $(5.3)$ and a regularizer $\tilde{E}_{\infty}(t, s)$ Q.E.D.

\section{\$6. Construction of the Furndanemtal Solution for a Hyperbolic System} (Proof of Theorem 3)

In this section, we construct the fundamental solution of the system (4.20). First, we apply Proposition 5.1 to each element of $D_{t}-\mathscr{D}(t)+F(t)$. Then, the fundamental solution $\mathbb{E}^{0}(t, s)$ of $D_{t}-\mathscr{D}(t)+\mathbb{F}(t)$ is constructed in the form 


$$
E^{0}(t, s)=\left(\begin{array}{cc}
I_{\phi_{+}}(t, s) & 0 \\
0 & I_{\phi_{-}}(t, s)
\end{array}\right)\left(\begin{array}{cc}
\tilde{E}_{+}(t, s) & 0 \\
0 & \tilde{E}_{-}(t, s)
\end{array}\right)+\tilde{E}_{\infty}(t, s)
$$

where $\tilde{E}_{ \pm}(t, s)$ are pseudo-differential operators with the symbols in $S_{\rho, G(\kappa)}\left[w_{0}\right]$ with $w_{0}(\theta)$ in $(5.3)$ and $\widetilde{E}_{\infty}(t, s)$ is a regularizer in $\mathscr{R}_{G(\kappa)}$. We seek the fundamental solution $E(t, s)$ of $(4.20)$ in the form

$$
E(t, s)=E^{0}(t, s)+\int_{s}^{t} E^{0}\left(t, t^{\prime}\right) V\left(t^{\prime}, s\right) d t^{\prime} .
$$

Then, $V(t, s)$ must satisfy

$$
P_{\phi}(t, s)-i V(t, s)+\int_{s}^{t} P_{\phi}\left(t, t^{\prime}\right) V\left(t^{\prime}, s\right) d t^{\prime}=0,
$$

where

$$
P_{\phi}(t, s)=\left(R(t)+R_{\infty}(t)\right) E^{0}(t, s) .
$$

Set

$$
\left\{\begin{array}{l}
V_{1}(t, s)=-i P_{\phi}(t, s), \\
V_{v+1}(t, s)=-i \int_{s}^{t} P_{\phi}\left(t, t^{\prime}\right) V_{v}\left(t^{\prime}, s\right) d t^{\prime} \quad(v \geqq 1) .
\end{array}\right.
$$

Then, we can get formally the solution $V(t, s)$ of $(6.2)$ in the form $V(t, s)=$ $\sum_{v=1}^{\infty} V_{v}(t, s)$.

Now, we estimate $V_{v+1}(t, s)$ in (6.3). From $(6.3) V_{v+1}(t, s)$ for $v \geqq 1$ has the form

$$
V_{v+1}(t, s)=(-i)^{v+1} \int_{s}^{t} \int_{s}^{t_{1}} \ldots \int_{s}^{t_{v-1}} P_{\phi}\left(t, t_{1}\right) P_{\phi}\left(t_{1}, t_{2}\right) \ldots P_{\phi}\left(t_{v}, s\right) d t_{v} \ldots d t_{1} .
$$

As in Section 5 we will consider a main part of $V_{v+1}(t, s)$. Then, modulo regularizers, $V_{v+1}(t, s)$ is equal to the sum of operators of the form

$$
\begin{aligned}
V_{v+1}^{1}(t, s)= & (-i)^{v+1} \int_{s}^{t} \int_{s}^{t_{1}} \ldots \int_{s}^{t_{v-1}} r_{1}\left(t, X, D_{x}\right) I_{\phi_{1}}\left(t, t_{1}\right) \\
& \times \tilde{e}_{1}\left(t, t_{1} ; X, D_{x}\right) r_{2}\left(t_{1}, X, D_{x}\right) I_{\phi_{2}}\left(t_{1}, t_{2}\right) \\
& \times \tilde{e}_{2}\left(t_{1}, t_{2} ; X, D_{x}\right) \ldots r_{v+1}\left(t_{v}, X, D_{x}\right) \\
& \times I_{\phi_{v+1}}\left(t_{v}, s\right) \tilde{e}_{v+1}\left(t_{v}, s ; X, D_{x}\right) d t_{v} \ldots d t_{1} .
\end{aligned}
$$

Here $\phi_{j}(t, s ; x, \xi)$ are $\phi_{+}(t, s ; x, \xi)$ or $\phi_{-}(t, s ; x, \xi)$ in Lemma $3.4, r_{j}(t, x, \xi)$ are symbols in $\mathscr{H}_{1, \delta, G(\kappa)}[\sigma, \omega]$ and $\tilde{e}_{j}\left(t_{j-1}, t_{j} ; x, \xi\right)$ are symbols in $S_{\rho, G(\kappa)}\left[w_{j}\right]$ with 


$$
\begin{gathered}
w_{j}(\theta)=\exp \left[C \theta^{\sigma} \log \left\{\left(t_{j-1} \theta^{\omega(1-\sigma)}+1\right) /\left(t_{j} \theta^{\omega(1-\sigma)}+1\right)\right\}\right] \\
\left(t_{0}=t, t_{v+1}=s\right) .
\end{gathered}
$$

Since $r_{j+1}\left(t_{j}, x, \xi\right) \in \mathscr{H}_{1, \delta, G(\kappa)}[\sigma, \omega] \subset S_{1, \delta, G(\kappa)}[\sigma, 0,-1]$ it follows that $\tilde{e}_{j}\left(t_{j-1}, t_{j} ; X, D_{x}\right) r_{j+1}\left(t_{j}, X, D_{x}\right)$ is a pseudo-differential operator with a main symbol in $S_{1, \delta, G(\kappa)}\left[w_{j}^{1}\right]$, where

$$
w_{j}^{1}(\theta)=\theta^{\sigma}\left(t_{j}+\theta^{-\omega(1-\sigma)}\right)^{-1} w_{j}(\theta) .
$$

Set $\Phi_{j, v+1}=\phi_{j}\left(t_{j-1}, t_{j}\right) \# \cdots \# \phi_{v+1}\left(t_{v}, s\right)$ and $\Phi_{v+1, v+1}=\Phi_{v+1}\left(t_{v}, s\right)$. Then, if we assume $0 \leqq s \leqq t \leqq T_{0}$, we have $\phi_{j} \in \mathscr{P}_{G(\kappa)}\left(\tilde{c} T_{0}\right)$ and $\Phi_{j, v+1} \in \mathscr{P}_{G(\kappa)}\left(\tilde{c} T_{0}\right)$ for a constant $\tilde{c}$. Take $T_{0}$ such that $T_{0} \leqq \tau^{0} /(2 \tilde{c})$ for a constant $\tau^{0}$ in Proposition 2.4. Then, we can apply Proposition 2.4 to find symbols $p_{j}^{1}(x, \xi) \equiv p_{j}^{1}\left(t_{j-1}, \ldots, t_{v}, s\right.$; $x, \xi)$ and $\tilde{r}_{j}^{1}(x, \xi) \equiv \tilde{r}_{j}^{1}\left(t_{j-1}, \ldots, t_{v}, s ; x, \xi\right)$ such that

$$
p_{j}^{1}(x, \xi) \in S_{\rho, G(\kappa)}\left[w_{j, c}^{1}\right] \quad \text { with } \quad w_{j, c}^{1}(\theta)=w_{j}^{1}(c \theta)
$$

for a constant $c(\geqq 1), r_{j, \infty}^{1}(x, \xi) \in \mathscr{R}_{G(\kappa)}$ and

$$
\begin{gathered}
I_{\phi_{j}}\left(t_{j-1}, t_{j}\right) \tilde{e}_{j}\left(t_{j-1}, t_{j} ; X, D_{x}\right) r_{j+1}\left(t_{j}, X, D_{x}\right) I_{\Phi_{j+1, v+1}}=I_{\Phi_{j, v+1}} P_{j}^{1}+\mathbb{R}_{j, \infty}^{1} \\
(j=1, \ldots, v) .
\end{gathered}
$$

Hence, $V_{v+1}^{1}(t, s)$ is equal to

$$
\begin{aligned}
V_{v+1}^{2}(t, s)= & (-i)^{v+1} \int_{s}^{t} \int_{s}^{t_{1}} \ldots \int_{s}^{t_{v-1}} r_{1}\left(t, X, D_{x}\right) I_{\Phi_{v+1}} \\
& \times \mathbb{P}_{1}^{1} P_{2}^{1} \ldots P_{v}^{1} \tilde{e}_{v+1}\left(t_{v}, s ; X, D_{x}\right) d t_{v} \ldots d t_{1}
\end{aligned}
$$

modulo regularizers, where $\Phi_{v+1}=\Phi_{1, v+1}$.

Next, we use discussion in the proof of Lemma 2.5. Then, there exist symbols $p_{0}\left(t, \tilde{t}^{v}, s ; x, \xi\right) \equiv p_{0}\left(t, t_{1}, \ldots, t_{v}, s ; x, \xi\right)$ in $\mathscr{H}_{1, \delta, G(\kappa)}[\sigma, \omega]$ and $r_{0, \infty}^{1}\left(t, \tilde{t}^{v}, s ; x, \xi\right)$ in $\mathscr{R}_{G(\kappa)}$ such that

$$
r_{1}\left(t, X, D_{x}\right) I_{\Phi_{v+1}}=\mathbb{P}_{0, \Phi_{v+1}}+R_{0, \infty}^{1} .
$$

Now, we consider the Fourier integral operator $\mathbb{P}_{0, \Phi_{v+1}}$ as a pseudo-differential operator with a symbol

$$
p_{0}^{1}\left(t, \tilde{t}^{v}, s ; x, \xi\right)=p_{0}\left(t, \tilde{t}^{v}, s ; x, \xi\right) \exp \left[i\left(\Phi_{v+1}-x \cdot \xi\right)\right] .
$$

Let $\sigma^{\prime}$ be a real number satisfying (5), and assume that $T_{0}$ satisfies $T_{0} \leqq T_{1}$ for a constant $T_{1}$ in Lemma 3.6. Then, from Lemma 3.7 and $p_{0}\left(t, \tilde{t}^{v}, s ; x, \xi\right) \in$ $\mathscr{H}_{1, \delta, G(\kappa)}[\sigma, \omega]$, it follows that $p_{0}^{1}\left(t, \tilde{t}^{v}, s ; x, \xi\right)$ satisfies

$$
\left|p_{0(\beta)}^{1(\alpha)}\right| \leqq C M^{-|\alpha+\beta|} \alpha !^{\kappa}\left(\beta !^{\kappa}+\beta !^{\kappa(1-\delta)}\langle\xi\rangle^{\delta|\beta|}\right)\langle\xi\rangle^{-|\alpha|} \exp \left(-\varepsilon t^{l+1} \mu(x, \xi)+C\langle\xi\rangle^{\sigma^{\prime}}\right)
$$

for an $\varepsilon>0$. Here, the term $\langle\xi\rangle^{\sigma} \mu(x, \xi)^{\omega}$ is absorbed into $\exp \left(C\langle\xi\rangle^{\sigma^{\prime}}\right)$. Now, to each pseudo-differential operator $P_{j}^{1}, j=0, \ldots, v$, we assign a 
pseudo-differential operator $P_{j}^{2} \quad$ with the symbol $\left\{\left(1-\Delta_{\xi}\left(\langle\xi\rangle^{2 \delta} \cdot\right)(1\right.\right.$ $\left.\left.+\langle\xi\rangle^{2 \delta}\left|x-x^{\prime}\right|^{2}\right)^{-1}\right\}^{[n / 2]+1} \sigma\left(P_{j}^{1}\right)$. Then, $V^{2}(t, s)$ is equal to

$$
V_{v+1}^{3}(t, s)=(-i)^{v+1} \int_{s}^{t} \int_{s}^{t_{1}} \ldots \int_{s}^{t_{v-1}} P_{0}^{2} P_{1}^{2} \ldots P_{v}^{2} \tilde{e}_{v+1}\left(t_{v}, s ; X, D_{x}\right) d t_{v} \ldots d t_{1}
$$

modulo regularizers. Let $\tilde{p}_{v+2}\left(t, \tilde{t}^{v}, s ; x, \tilde{\xi}^{v+1}, \tilde{x}^{v+1}, \xi\right)$ be a multiple symbol corresponding to $P_{0}^{2} P_{1}^{2} P_{2}^{2} \ldots P_{v}^{2} \tilde{e}_{v+1}\left(t_{v}, s ; X, D_{x}\right)$ and set

$$
\tilde{p}_{v+2}^{\prime}\left(t, s ; x, \tilde{\xi}^{v+1}, \tilde{x}^{v+1}, \xi\right)=\int_{s}^{t} \int_{s}^{t_{1}} \ldots \int_{s}^{t_{v}} \tilde{p}_{v+2}\left(t, \tilde{t}^{v}, s ; x, \tilde{\xi}^{v+1}, \tilde{x}^{v+1}, \xi\right) d t_{v} \ldots d t_{1} .
$$

Then, $\tilde{p}_{v+2}\left(t, \tilde{t}^{v}, s ; x, \tilde{\xi}^{v+1}, \tilde{x}^{v+1}, \xi\right)$ satisfies (1.20) with $v$ replaced by $v+1$ and $w_{v+1}\left(\max _{j}\left\langle\xi^{j}\right\rangle\right)$ replaced by $\tilde{w}_{v+2}\left(x, \max _{j}\left\langle\xi^{j}\right\rangle\right)$. Here, $\tilde{w}_{v+2}(x, \theta)$ $\left(=\tilde{w}_{v+2}\left(t, \tilde{t}^{v}, s ; x, \theta\right)\right)$ is defined by

$$
\tilde{w}_{v+2}(x, \theta)=\exp \left[-\varepsilon t^{l+1} \tilde{\mu}(x, \theta)+C \theta^{\sigma^{\prime}}\right]\left(\prod_{j=1}^{v} w_{j, c}^{1}(\theta)\right) w_{v+1}(\theta)
$$

for $\tilde{\mu}(x, \theta)=|g(x)|^{l^{\prime}} \theta^{1-\sigma}+1, w_{j, c}^{1}(\theta)$ in (6.6), $w_{v+1}(\theta)$ in (6.4) and positive constants $\varepsilon$ and $C$. From (6.4)-(6.5) we have

$$
\begin{aligned}
\prod_{j=1}^{v} w_{j, c}^{1}(\theta) w_{v+1}(\theta) \leqq & (c \theta)^{v \sigma} \prod_{j=1}^{v}\left(t_{j}+(c \theta)^{-\omega(1-\sigma)}\right)^{-1} \prod_{j=1}^{v+1} w_{j}(c \theta) \\
\leqq & (c \theta)^{v \sigma} \prod_{j=1}^{v}\left(t_{j}+(c \theta)^{-\omega(1-\sigma)}\right)^{-1} \\
& \times \exp \left[C(c \theta)^{\sigma} \log \left\{\left(t(c \theta)^{\omega(1-\sigma)}+1\right) /\left(s(c \theta)^{\omega(1-\sigma)}+1\right)\right\}\right]
\end{aligned}
$$

and

$$
\begin{aligned}
& \int_{s}^{t} \int_{s}^{t_{1}} \ldots \int_{s}^{t_{v-1}} \prod_{j=1}^{v}\left(t_{j}+(c \theta)^{\omega(1-\sigma)}\right)^{-1} d t_{v} \ldots d t_{1} \\
& \quad=\left\{\log \left\{\left(t(c \theta)^{\omega(1-\sigma)}+1\right) /\left(s(c \theta)^{\omega(1-\sigma)}+1\right\}\right\}^{v} / v ! .\right.
\end{aligned}
$$

Hence, setting

$$
\left\{\begin{aligned}
\tilde{w}_{v+2}^{1}(x, \theta)=\exp \left[-\varepsilon t^{l+1} \tilde{\mu}(x, \theta)+C \theta^{\sigma^{\prime}}\right] \tilde{\tilde{w}}_{v}(c \theta) / v ! \\
\tilde{\tilde{w}}_{v}(\theta)=\exp \left[C \theta^{\sigma} \log \left\{\left(t \theta^{\omega(1-\sigma)}+1\right) /\left(s \theta^{\omega(1-\sigma)}+1\right)\right\}\right] \\
\times\left\{\theta^{\sigma} \log \left\{\left(t \theta^{\omega(1-\sigma)}+1\right) /\left(s \theta^{\omega(1-\sigma)}+1\right)\right\}\right\}^{v}
\end{aligned}\right.
$$

$\tilde{p}_{v+2}^{\prime}\left(t, s ; x, \tilde{\xi}^{v+1}, \tilde{x}^{v+1}, \xi\right)$ satisfies $(1.20)$ with $v$ replaced by $v+1$ and $w_{v+1}\left(\max _{j}\left\langle\xi^{j}\right\rangle\right)$ replaced by $\tilde{w}_{v+2}^{1}\left(x, \max _{j}\left\langle\xi^{j}\right\rangle\right)$. Although $\tilde{w}_{v+2}^{1}(x, \theta)$ is not an ordered function, it satisfies (1.19) and, setting 


$$
\tilde{w}_{v+2}^{2}(x, \theta)=\exp \left[-\varepsilon t^{l+1} \tilde{\mu}(x, \theta / 2)+C(2 \theta)^{\sigma^{\prime}}\right] \tilde{\tilde{w}}_{v}(2 c \theta) / v !,
$$

$\tilde{w}_{v+2}^{1}(x, \xi)$ satisfies $\tilde{w}_{v+2}^{1}\left(x, \theta^{\prime}\right) \leqq \tilde{w}_{v+2}^{2}(x, \theta)$ when $\theta^{\prime} / 2 \leqq \theta \leqq 2 \theta^{\prime}$. Hence, we can use the discussion of proving Lemma 1.6 and we find that $V_{v+1}^{3}(t, s)$ is a sum of pseudo-differential operators $v_{v+1}^{3}\left(t, s ; X, D_{x}\right)$ and $v_{v+1, \infty}^{3}\left(t, s ; \mathbb{X}, \mathbb{D}_{x}\right)$ with symbols $v_{v+1}^{3}(t, s ; x, \xi)$ and $v_{v+1, \infty}^{3}(t, s ; x, \xi)$ satisfying

$$
\begin{aligned}
\mid v_{v+1(\beta)}^{3}(\alpha)(t, s ; x, \xi) \leqq & C^{v} \mathbb{M}^{-|\alpha+\beta|} v !^{-1} \\
& \times\left(|\alpha+\beta| !^{\kappa}+|\alpha+\beta| !^{\kappa \rho}\langle\xi\rangle^{(1-\rho)|\alpha+\beta|}\right)\langle\xi\rangle^{-|\alpha|} \\
& \times \exp \left[-\varepsilon t^{l+1}|g(x)|^{l^{\prime}}(\langle\xi\rangle / 2)^{(1-\sigma)}+C(2\langle\xi\rangle)^{\sigma^{\prime}}\right] \\
& \times \tilde{\tilde{w}}_{v}(2 c\langle\xi\rangle) \\
\leqq & C^{\prime v} \mathbb{M}^{-|\alpha+\beta|} v !^{-1} \\
& \times\left(|\alpha+\beta| !^{\kappa}+|\alpha+\beta| !^{\kappa \rho}\langle\xi\rangle^{(1-\rho)|\alpha+\beta|}\right)\langle\xi\rangle^{v \sigma^{\prime}-|\alpha|} \\
& \times \exp \left[-\varepsilon t^{l+1}|g(x)|^{l^{\prime}}\langle\xi\rangle^{(1-\sigma)} / 2+\mathbb{C}^{\prime}\langle\xi\rangle^{\sigma^{\prime}}\right], \\
\mid v_{v+1, \infty}^{3}(\alpha) & (t, s ; x, \xi) \mid \leqq C^{v} C_{\alpha} \mathbb{M}^{-|\beta|} v !^{-1+\sigma^{\prime} \kappa} \beta !^{\kappa} \exp \left(-\varepsilon\langle\xi\rangle^{1 / \kappa}\right) .
\end{aligned}
$$

Here, we used $\sigma<\sigma^{\prime}$ in (6.7). Summing up, we can prove that modulo regularizers $V_{v+1}(t, s)$ is equal to a pseudo-differential operator $\mathbb{V}_{v+1}^{0}(t, s)$ whose symbol satisfies the similar estimate to (6.7). We can also prove that $\mathbb{V}_{v+1}(t, s)-$ $V_{v+1}^{0}(t, s)$ is a pseudo-differential operator with a symbol satisfying (6.8).

From the above discussion we can prove that the operator

$$
\int_{s}^{t} E^{0}\left(t, t^{\prime}\right) V\left(t^{\prime}, s\right) d t^{\prime}
$$

in (6.1) can be written in the form

$$
E_{0}(t, s)+E_{\infty}(t, s)
$$

with symbols $e_{0}(t, s ; x, \xi)$ and $e_{\infty}(t, s ; x, \xi)$ satisfying (11) and (12), respectively. We note that by $\sigma<\sigma^{\prime}$ the operator $\mathbb{E}^{0}(t, s)$ can be written (modulo regularizers) in the form

$$
\mathbb{I}_{\phi_{+}} E_{+}(t, s)+\mathbb{I}_{\phi_{-}} E_{-}(t, s)
$$

with pseudo-differential operators $\mathbb{E}_{ \pm}(t, s)$ whose symbols satisfy $(10)$. Consequently, we have proved Theorem 3 .

\section{Referemees}

[1] Alinhac, S., Paramétrix et propagation des singularités pour un problème de Cauchy à multiplicité variable, Soc. Math. France Astérisque, 34-35 (1976), 3-36.

[2] Aoki, T., Symbols and formal symbols of pseudodifferential operators, Advanced Studies Pure Math., \& (1984), 181-208. 
[3] Boutet de Monvel, L., Hypoelliptic operators with double characteristics and related pseudodifferential operators, Comm. Pure Appl. Math., 27 (1974), 585-639.

[4] Boutet de Monvel, L. and Krée, P., Pseudo-differential operators and Gevrey class, Ann. Inst. Fourier, Grenoble, 17 (1967), 295-323.

[5] Cattabriga, L. and Zanghirati, L., Fourier integral operators of infinite order on Gevrey spaces. Applications to the Cauchy problem for hyperbolic operators, Proc. NATO A.S.I. on "Advances in Microlocal Analysis" I1 Ciocco, Italy, September 2-12, 1985, to appear.

[6] Hashimoto, S., Matsuzawa, T. and Morimoto, Y., Opérateurs pseudodifférentiels et classes de Gevrey, Comm. Partial Differential Equations, 8 (1983), 1277-1289.

[7] Hörmander, L., Uniqueness theorems and wave front sets for solutions of linear partial differential equations with analytic coefficients, Comm. Pure Appl. Math., 24 (1971), 671-704.

[8] Itho, S. and Uryu, H., Conditions for well-posedness in Gevrey classes of the Cauchy problems for Fuchsian hyperbolic operators II, preprint.

[9] Ivrii, V. Ja., Cauchy problem conditions for hyperbolic operators with characteristics of variable multiplicity for Gevrey classes, Siberian Math. J., 17 (1976), 921-931.

[10] Iwasaki, C., Gevrey-hypoellipticity and pseudo-differential operators on Gevrey class, Pseudo-Differential Operators, Lecture note in Math., 1256 (1987), 281-293.

[11] Kumano-go, H., Fundamental solution for a hyperbolic system with diagonal principal part, Comm. Partial Differential Equations, 4 (1979), 959-1015.

[12] — , Pseudo-differential operators, (The MIT Press, Cambridge and London, 1982).

[13] Kumano-go, H., Taniguchi, K. and Tozaki, Y., Multi-products of phase functions for Fourier integral operators with an application, Comm. Partial Differential Equations, 3 (1978) 349-380.

[14] Métivier, G., Analytic hypoellipticity for operators with multiple characteristics, Comm. Partial Differential Equations, 6 (1982), 1-90.

[15] Morimoto, Y. and Taniguchi, K., Propagation of wave front sets of solutions of the Cauchy problem for hyperbolic equations in Gevrey classes, Osaka J. Math., 23 (1986), 765-814.

[16] Rodino, L. and Zanghirati, L., Pseudo differential operators with multiple characteristics and Gevrey singularities, Comm. Partial Differential Equations, $\mathbb{1} \mathbb{1}$ (1986), 673-711.

[17] Shinkai, K., On the fundamental solution for a degenerate hyperbolic system, Osaka J. Math., 18 (1981), 257-288.

[18] — Branching of singularities for a degenerate hyperbolic system, Comm. Partial Differential Equations, 7 (1982), 581-607.

[19] —, Gevrey wave front sets of solutions for a weakly hyperbolic operator, Math. Japon., 30 (1985), 701-717.

[20] —, Stokes multipliers and a weakly hyperbolic operator, University of Minnesota, Mathematics Report, \#86-134, (1987).

[21] Taniguchi, K., Multi-products of Fourier integral operators and the fundamental solution for a hyperbolic system with involutive characteristics, Osaka J. Math., 21 (1984) 169-224.

[22] - Fourier integral operators in Gevrey class on $R^{n}$ and the fundamental solution for a hyperbolic operator, Publ. RIMS, Kyoto Univ., 20 (1984), 491-542.

[23] — Pseudo-differential operators acting on ultradistributions, Math. Japon., 30 (1985), 719-741.

[24] Taniguchi, K. and Tozaki, Y., A hyperbolic equation with double characteristics which has a solution with branching singularities, Math. Japon., 25 (1980), 279-300.

[25] Zanghirati, L., Pseudodifferential operators of infinite order and Gevrey classes, Ann. Univ. Ferrara-Sez. VII-Sc. Mat., 31 (1985), 197-219. 
